\title{
eJRIEPS
}

Ejournal de la recherche sur l'intervention en éducation physique et sport

Hors-série $N^{\circ} 1 \mid 2015$

Quelques aspects théoriques de la didactique des sports collectifs. Préparation aux concours de recrutement

\section{L'apport de la notion de configuration du jeu pour analyser les rapports d'opposition}

Jean-Francis Gréhaigne, Patrick Marle et Didier Caty

\section{OpenEdition}

\section{Journals}

Édition électronique

URL : http://journals.openedition.org/ejrieps/1277

DOI : $10.4000 /$ ejrieps. 1277

ISSN : 2105-0821

Éditeur

ELLIADD

Référence électronique

Jean-Francis Gréhaigne, Patrick Marle et Didier Caty, «L'apport de la notion de configuration du jeu pour analyser les rapports d'opposition », eJRIEPS [En ligne], Hors-série Nº 1 | 2015, mis en ligne le 01 décembre 2015, consulté le 04 octobre 2019. URL : http://journals.openedition.org/ejrieps/1277 DOI : 10.4000/ejrieps. 1277

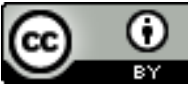

La revue eJRIEPS est mise à disposition selon les termes de la Creative Commons Attribution 4.0 International License. 
eJRIEPS Hors série n¹ Décembre 2015

\title{
L'apport de la notion de configuration du jeu
} pour analyser les rapports d'opposition

\author{
Jean-Francis Gréhaigne*, Patrick Marle ${ }^{* *}$ \& Dider Caty ${ }^{* * *}$ \\ * Professeur des Universités retraité, Université Bourgogne Franche-Comté \\ ** Professeur Agrégé d'EPS retraité, Université Bourgogne Franche-Comté \\ ${ }^{* *}$ Professeur Agrégé d'EPS, Lycée Cournot Gray
}

Cet article se veut une note de synthèse à propos du concept de configuration du jeu en sport collectif. II tente de rassembler différents écrits dispersés dans diverses contributions (Gréhaigne, 2007 ; Gréhaigne, Caty, \& Marle, 2004 ; Gréhaigne, Marle, \& Zerai, 2013). Au plan général, on utilise l'expression configuration d'un match pour désigner le caractère particulier que revêt une confrontation entre deux équipes. Elle énonce l'architecture et les éléments constituants de l'opposition (ses aspects d'ordre physique, psychologique, social...). La définition la plus courante de configuration (du latin configurare) consiste à "donner une forme " ou "forme extérieure d'un ensemble » ou encore «la disposition relative ou l'arrangement des parties ou des éléments d'une chose ». En sport collectif, on distingue habituellement les configurations momentanées du jeu et les configurations perçues par le joueur. A ce propos, Paillard (1987) affirme que

L'apprentissage perceptif consiste à extraire des schèmes de configurations d'indices pertinents caractéristiques, dont la covariance ou la co-présence, dans une situation donnée, permet de réduire le temps d'analyse et d'évolution du contexte informationnel, par le choix d'indicateurs privilégiés, prédictifs de la situation globale. Cela permet (...) " l'identification de structures d'espaces propres à rendre compte de l'étonnante capacité de notre système nerveux à détecter les invariants et les régularités. Ce qui nous confronte au problème de l'identification des critères qui déterminent le choix parmi les directions possibles que peut prendre le processus organisationnel, de celles qui seront retenues et stabilisées (p. 1422).

Gréhaigne, Godbout \& Bouthier (1997, 2001) proposent que ce concept de configuration perçue du jeu se trouve au cœur de la réflexion didactique à propos des décisions prises en jeu. II renvoie aux positions relatives des joueurs des deux équipes, en relation avec la possession et la position du ballon, ainsi qu'avec les déplacements des joueurs. Ce concept se décline sous plusieurs acceptions et s'apparente aux notions de «patterns of play " (Ali \& Farraly, 1990), "situation of play (Mc Pherson, 1993), "display " (Mc Morris \& Graydon, 1997) et de "spatial configuration» (Hemphill, 2005). L'étude des 


\section{eJRIEPS Hors série n¹ Décembre 2015}

configurations du jeu nous paraît importante car elle est susceptible d'optimiser l'activité des joueurs durant le jeu de mouvement, en leur permettant de caractériser des états momentanés du rapport de forces et leurs évolutions probables. Ainsi, une analyse pertinente des configurations du jeu aide à comprendre l'évolution du jeu même si le recours à un schéma prévu à l'avance, peut constituer une adaptation tactique temporairement efficace.

Deleplace (1979) parle, lui, de " constellation de joueurs ", entendant par-là l'ensemble des joueurs (appartenant à l'une ou l'autre équipe) qui sont effectivement impliqués, à un moment donné, dans une « unité tactique » en cours. En ce qui concerne l'attaque, cette notion est très importante pour mettre en exergue le fait que, pour tout joueur s'apprêtant à intervenir directement sur le ballon à un moment quelconque du mouvement, il est indispensable qu'il se représente bien le rapport d'opposition momentané. C'est-à-dire qu'il doit tenir compte de tous les adversaires et aussi tous les partenaires qu'il faut inventorier dans son environnement proche car ils ne sont pas tous forcément en mesure, à l'instant considéré, de prendre une part efficace à l'action en train de se dérouler. Les raisons de cette carence peuvent être multiples : soit un placement relatif momentané inadéquat, soit une orientation temporairement défavorable de leur direction de course, soit une vitesse trop importante ou un manque de vitesse, soit une direction de course inadéquate, soit encore un rapport d'appuis défavorable, soit plusieurs de ces causes en même temps. En fait, c'est comme s'ils n'étaient pas là dans le système " affrontement partiel ». II est ainsi déterminant que tout joueur soit capable d'estimer ce qu'est la configuration réelle des joueurs capables d'intervenir dans l'environnement proche, c'està-dire l'effectif réel des acteurs possibles, à l'exclusion de ceux déjà consommés ou hors de position. La construction de ces conduites exploratoires dans le jeu, conduit le joueur à pouvoir détecter, identifier et exploiter un nombre significatif de constellations caractéristiques de ce qui doit être et, peut être effectivement entrepris dès le tout premier instant de l'initiation. En effet, un grand problème de la pratique des sports collectifs est l'acquisition de cette capacité à voir et analyser la configuration présente dans laquelle on se trouve engagé personnellement. Ce constat est valable pour le porteur de balle mais aussi pour ceux qui l'entourent, car c'est à eux de proposer des solutions dans l'espace proche et lointain pour favoriser l'enchaînement du mouvement collectif.

Pendant le jeu, une suite de configurations du jeu se développe d'un état 1 à un état 2 , etc. jusqu'à un état $n$ tant que la balle reste en jeu. II y a deux façons d'appréhender les phénomènes. D'abord, comme dans une photographie, la configuration de jeu peut être 


\section{eJRIEPS Hors série n¹ Décembre 2015}

définie par les positions des joueurs à un temps t (Gréhaigne, Bouthier, \& David, 1997). Ceci mène à une étude bidimensionnelle statique de la distribution spatiale des attaquants et des défenseurs et de l'emplacement de la balle. Considérant alors la suite des configurations comme une série d'images, on pourrait amener à déterminer les raisons des choix des attaquants et défenseurs.

Cependant, une autre façon de considérer le problème de façon dynamique consiste à déterminer chaque micro-état par la distribution des joueurs et de la balle sur le terrain à partir de leurs emplacements respectifs, des orientations par rapport à la direction du jeu et des vitesses de déplacement (Gréhaigne, Bouthier, \& David, 1997). La prise en compte de telles configurations dynamiques du jeu représente une réponse plus élaborée pour en décrire la réalité car les rapports d'opposition sont pris en compte dans leur continuité et aussi parce que les séquences de jeu s'engendrent les unes dans les autres. La reconnaissance et l'exploitation de ces configurations dynamiques, dont certaines peuvent être prometteuses, sont à effectuer, au fil de l'apprentissage, dans des rapports d'opposition de plus en plus denses. La complexité et l'incertitude reposent ici sur le temps et la vitesse à disposition du joueur pour décider et faire. Temps et vitesse deviennent donc des éléments cruciaux de la réussite et le cadre de référence nécessaire à toutes décisions. Avec ce rapport au temps, nous pouvons identifier différents concepts : « avance », « retard », « jouer au temps juste », « vitesse de transmission du ballon » (Dugrand, 1989), « temps de jeu », etc. En définitive, au fil des progrès des élèves rien ne peut être fondamentalement compris en sport collectif si l'on ne substitue pas à un référentiel spatial, un référentiel temporel permettant de traiter de façon plus pertinente les informations à disposition. Dans ce cas, l'espace ne devient qu'un indicateur, certes privilégié, d'un rapport d'opposition, mais le temps dont dispose un joueur ou un groupe de joueurs pour agir ou pour parcourir un espace est, à notre sens, l'élément fondamental majeur pour comprendre l'évolution de ce rapport de forces et des configurations momentanées du jeu.

Configuration perçue, constellation, configuration momentanée du jeu nous semblent se rapporter à un même ensemble cohérent pour mieux analyser le jeu. Face à ces configurations, la tactique individuelle permet aux joueurs de répondre aux problèmes posés par l'évolution probable du jeu. Cette tactique individuelle combine, au sein de la perception, les compétences motrices développées tout au long de la vie, les connaissances tactiques qui y sont rattachées. Cela permet au porteur de balle de décider la manière de poursuivre le jeu. La visée dans toute formation de joueur est d'améliorer 


\section{eJRIEPS Hors série n¹ Décembre 2015}

chez lui le choix et la pertinence de ses réponses au sein des différentes situations de jeu. Un jeu de qualité chez les joueurs consiste à utiliser ses points forts pour marquer mais aussi à tirer parti des faiblesses de l'adversaire ainsi que saisir toutes les occasions qui se présentent : en un mot être compétent.

Avec l'objectif de développer ces éléments, cet article revient d'abord sur la dynamique du jeu et les problèmes posés par les perceptions et les prises de décisions à propos des configurations du jeu. Ensuite, l'étude de différentes circulations du ballon permet la mise en évidence de configurations prototypiques. Enfin, l'étude de quelques concepts complémentaires et une discussion sont menées en espérant apporter, aux élèves et aux enseignants, une aide pratique à l'observation et au décodage du jeu.

\section{Dynamique du jeu}

Une rencontre de sport collectif, constitue un système complexe où la finalité (organisation en fonction d'un but) est le gain du match. L'étude des interactions entre joueurs, partenaires ou adversaires, constitue l'élément décisif pour la pertinence de toute analyse. Ainsi, comprendre l'organisation du jeu et sa logique constitue un objectif fondamental de toute étude. Cette notion d'organisation impose, en particulier dans l'examen des configurations du jeu, la description de niveaux d'organisation avec l'idée sous-jacente d'une complexité à étudier. Ainsi, dans une rencontre de football, nous avons identifié plusieurs systèmes emboîtés constituant autant de niveaux d'organisation en interaction (Gréhaigne, 1989, 1992 ; Gréhaigne, Bouthier \& David, 1997 ; Gréhaigne, Godbout \& Bouthier, 1999) produisant des configurations particulières :

- le niveau d'organisation « match »;

- le niveau d'organisation « équipe »;

- le niveau d'organisation « affrontement partiel »;

- le niveau d'organisation « 1 contre 1 »;

- le niveau d'organisation « joueur ».

Concernant spécifiquement les effets des rapports d'opposition dans le déroulement d'une quelconque séquence de jeu, nous distinguerons trois niveaux d'affrontement différents (Gréhaigne, 1989). Tout d'abord ce que nous avons appelé le système " match », qui comprend l'effectif total des deux équipes. Ensuite, le système " affrontement partiel », qui oppose des sous-groupes pouvant aller d'une simple doublette jusqu'à la moitié de l'effectif de chaque équipe ; enfin le système " 1 contre 1 ", qui recouvre les effets du duel attaquant/défenseur (figure 1). Bien sûr, les effets s'engendrent les uns les autres et 


\section{eJRIEPS Hors série n¹ Décembre 2015}

rejaillissent à la fois dans la simultanéité de l'instant et dans la succession des instants. Cela amène à considérer la modélisation du jeu dans le temps comme un facteur clef de l'analyse du jeu.

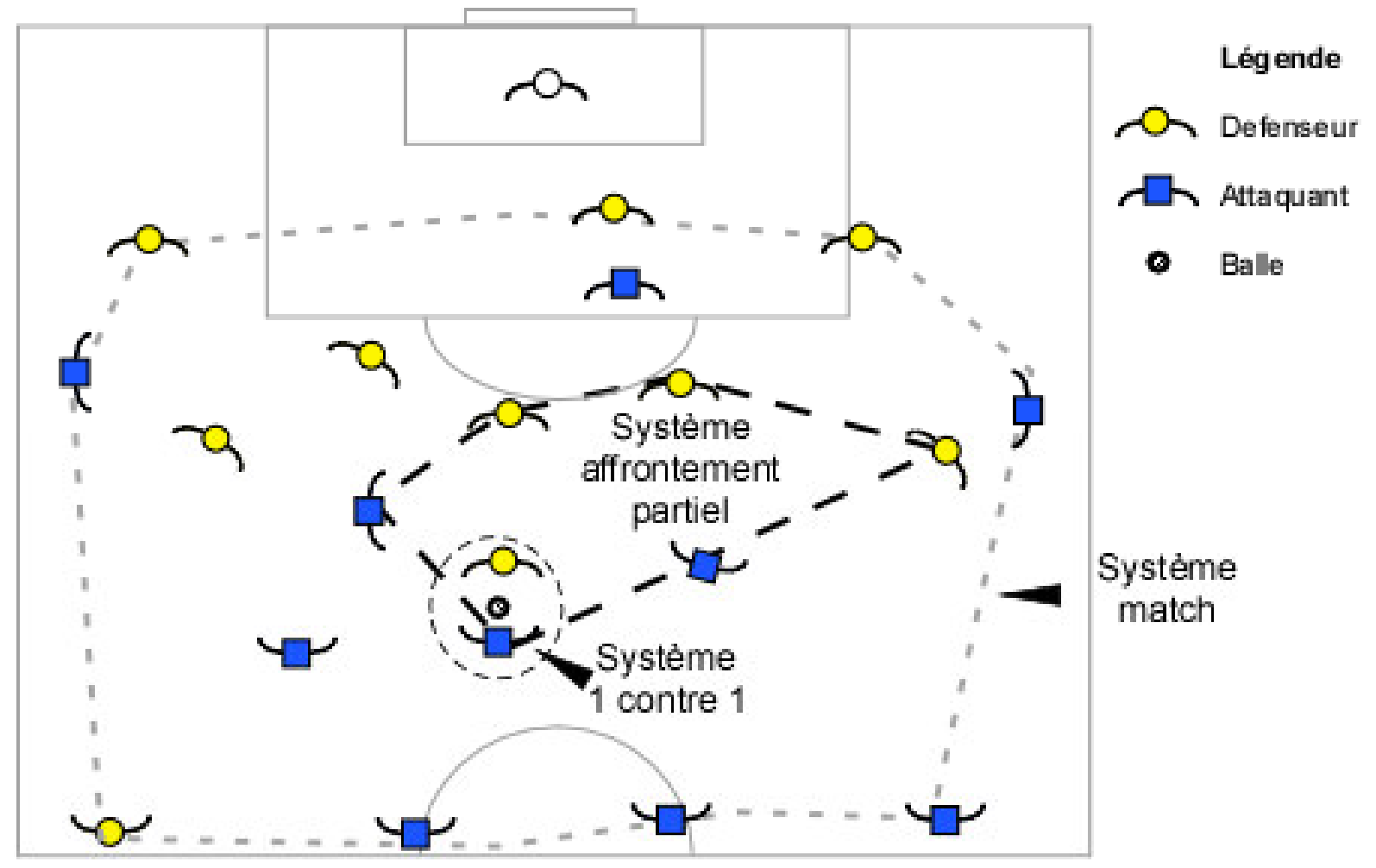

Figure 1. Trois niveaux d'organisation de systèmes emboîtés d'affrontement dans le jeu.

Pour affiner cette conception de l'analyse du jeu, basée, comme nous l'avons vu, sur le mouvement dans les systèmes dynamiques (Gréhaigne \& Godbout, 2014), nous allons utiliser comme outil d'observation l'espace de jeu effectif (EJE) et ses dérivés qui constituent des instruments faciles à manier et qui permettent d'extraire des données fiables des configurations du jeu.

Cet outil d'observation apparaît pour la première fois dans les études réalisées par des enseignants d'éducation physique réunis au sein du Conseil Pédagogique et Scientifique de la Fédération Sportive et Gymnique du Travail, à propos de l'observation des jeux sportifs collectifs aux Jeux Olympiques de Montréal en 1976 (Mérand, 1977). On peut définir l'EJE ainsi :

- le système de repères propre à définir l'espace de jeu effectif est constitué par l'ensemble des lignes qui délimitent l'aire de jeu réglementaire ;

- les variables observables sont les positions des joueurs situés, à l'instant $T$, à la périphérie des équipes (les gardiens de but n'étant pas pris en compte); 
eJRIEPS Hors série n¹ Décembre 2015

- ces positions, lorsqu'on les relie, délimitent une surface polygonale appelée par définition l'espace de jeu effectif (noté EJE).

Cela constitue les propriétés synchroniques de l'espace de jeu effectif. Cette conception de l'EJE postule qu'entre les éléments des deux équipes en situation d'opposition compétitive s'opère une structuration des relations de coopération et d'opposition. Compte tenu de la position de l'EJE sur le terrain et de la direction de l'attaque, la position des joueurs des deux équipes et du ballon constitue une configuration du jeu particulière à l'instant T. Dans ces conditions, l'EJE de la configuration définie et son éventuelle évolution permettent d'obtenir des informations significatives sur la valeur et les limites de l'adaptation, du joueur, c'est-à-dire sur ce que son comportement exprime de novateur, de créatif, ou au contraire de «régressif» dans sa lutte contre ses adversaires. Les propriétés diachroniques de l'EJE sont constituées par la succession des configurations momentanées du jeu ainsi définies par ses transformations dans le temps (ses emplacements, ses déformations, ses déplacements) dans le champ de jeu tout cela fournissant une première série d'indicateurs sur l'évolution du rapport de force entre les équipes au cours du match.

Cette surface est différente de l'aire de jeu, telle que délimitée par le règlement du sport en question. L'espace de jeu effectif, selon le jeu sportif collectif et la phase de jeu envisagée, peut être plus petit, équivalent ou plus grand que l'aire de jeu comme au volley-ball par exemple. Dans le jeu en mouvement en football, les gardiens de but ne sont pas inclus dans l'EJE.

Les transformations de cette surface polygonale constituent des observables fiables pour l'évolution des rapports topologiques entre les joueurs d'une même équipe avec les trajets et les trajectoires successives du ballon. II est alors possible de mieux caractériser l'organisation d'une équipe en attaque ou en défense pour une phase de jeu. Cette organisation est précisée en termes de jeu à la périphérie de l'EJE, de la périphérie à la zone centrale ou de la zone centrale vers la périphérie. L'élargissement ou le rétrécissement de l'espace de jeu sont à envisager du point de vue de la circulation des joueurs, de leur rayonnement personnel (en référence au débutant) et du point de vue de la circulation du ballon.

Ensuite, il est possible de définir l'espace de jeu effectif offensif (EJEO) et l'espace de jeu effectif défensif (EJED) à partir de l'espace de jeu effectif. On obtient alors deux surfaces polygonales plus ou moins interpénétrées. Les relations qui s'établissent entre ces deux espaces antagonistes et leurs évolutions respectives dans le temps doivent permettre 


\section{eJRIEPS Hors série n¹ Décembre 2015}

d'apporter des précisions sur les évolutions du rapport de forces durant les matchs. En particulier, la position du ballon ou du porteur de balle dans l'espace de jeu effectif offensif en fonction des phases de l'attaque donne de bonnes indications avec le ballon à l'arrière, à l'intérieur, à la périphérie ou à l'avant de l'espace de jeu effectif offensif (Gréhaigne, Godbout, \& Zerai, 2010). La figure 2a illustre la position d'un ballon à l'arrière de l'espace de jeu effectif et la figure $2 b$ décompose cet EJE en deux sous-ensembles EJEO et EJED. A partir de ces données, on dira qu'une défense en barrage est positionnée entre le ballon et sa propre cible, alors qu'une défense à la poursuite est une défense momentanément hors de position qui cherche à se repositionner, le plus rapidement possible entre le ballon et le but. Dans ce dernier cas, l'attaque possède de l'avance sur le replacement défensif. Quant à l'espace de jeu occupé (EJo), il est constitué par une succession d'EJE qui représente une surface polygonale révélant l'espace de jeu dynamique occupé par les attaquants et les défenseurs le temps d'une attaque. C'est l'espace maximum utilisé lors d'une séquence de jeu avec éventuellement une succession de déformations qui montre la façon dont cet espace a été parcouru. Cet outil diachronique devient alors intéressant pour observer l'élasticité et les déformations (contraction et expansion) au cours du temps des différents EJE qui se succèdent (sur ce thème, voir Gréhaigne \& Godbout, 2012). Enfin, toujours dans une perspective dynamique en sport collectif, la compression et/ou l'extension de l'EJEO et de l'EJED fonctionnent en étroite symbiose. Leurs analyses permettent aux joueurs de décrypter et/ou d'anticiper le mouvement dans le jeu en vue de prendre des décisions judicieuses. Avec des effectifs un peu plus importants, la zone d'intersection de ces deux espaces est une zone particulièrement sensible voire instable, à la fois pour l'équipe qui possède la balle car elle peut être mise en difficulté dans sa relance mais aussi pour la défense qui peut, avec un replacement intelligent, espérer effectuer une récupération haute du ballon. 


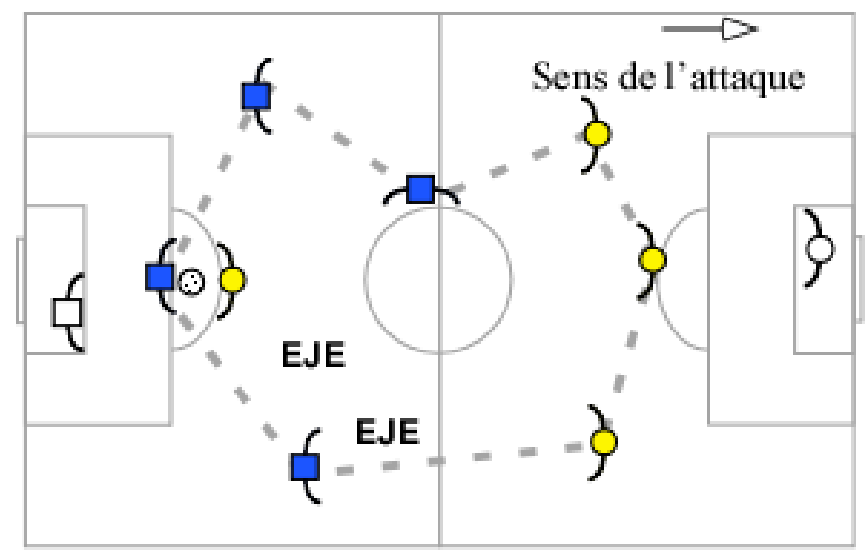

Figure 2a. Espace de jeu effectif et position du ballon à l'AR de l'EJE.

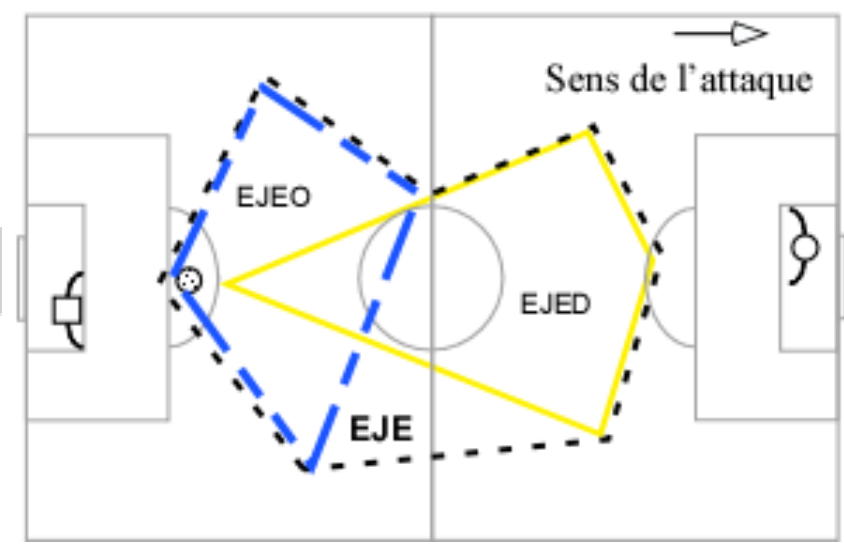

Figure 2b. Espace de jeu effectif décomposé en EJEO (noir) et EJED (gris) en barrage.

Les transitions entre deux configurations sont aussi porteuses d'informations notables sur l'évolution du jeu. Ainsi, l'évolution du système peut alors se modéliser en concevant une évolution discontinue dans le temps à partir de la mise en relation de cinq critères :

- position sur le terrain et volume de l'espace de jeu effectif (EJE);

- position et trajectoire du ballon ;

- positions respectives de l'espace de jeu effectif offensif (EJEO) et de l'espace de jeu effectif défensif (EJED) ainsi que leurs dynamiques d'évolution ;

- positions de la défense : en barrage ou à la poursuite ;

- l'extension ou la compression de l'EJE dans une suite de configurations qui permet d'obtenir une représentation assez fiable du rapport d'opposition.

Ici, l'incertitude constitue donc une notion importante qui est fondamentalement liée au temps. Un prélèvement rapide et précis d'informations simples rend possible la réduction d'incertitude. Sur le plan tactique, chaque équipe a, dès lors, pour objectif de réduire le désordre pour elle tout en l'augmentant pour l'équipe adverse.

La perception de cette dynamique, n'est pas simple à mettre en œuvre. Effectivement, la transmission d'un événement à travers un système d'interfaces est forcément incomplète du fait de la perte d'informations. Elle aboutit nécessairement à une configuration subjective. II en résulte une perte considérable dans la transmission de l'information mais ce phénomène est paradoxalement positif. C'est, en effet, l'indétermination partielle des structures qui est source de transformation. Le gain en plasticité, souplesse, aisance d'adaptation, possibilité de progrès et de développement..., compense de beaucoup ce 


\section{eJRIEPS Hors série n¹ Décembre 2015}

qui est perdu en transmission stricte d'informations. Toute interprétation cognitive devient alors une sorte d'enjeu au cours duquel le joueur introduit une détermination subjective pour combler les indéterminations liées à la plasticité. A l'inverse, c'est parce que cette détermination subjective est en partie erronée qu'une correction ultérieure est possible.

\section{Analyse de la situation de jeu : perception et conscience de la configuration}

La modélisation théorique développée par Ochanine (1978) dans le cadre de la conscience de la situation nous paraît très adaptée à la perception des configurations du jeu avec des joueurs forcément à rationalité limitée. On retrouve ce type de conception dans les travaux de Bailly (2004) sur la conscience d'une situation chez un conducteur en circulation automobile. Dans ce projet, l'expression « réseau dynamique local » a été définie afin d'analyser les relations entre les voitures à un moment donné. Cette situation s'applique énormément à la modélisation théorique développée en sport collectif. Par ailleurs, Bisseret (1995), quant à lui, examine les représentations et les décisions expertes chez les aiguilleurs du ciel avec pour base le concept «mémoire opérationnelle » dont le contenu volatil reposerait sur l'image ou la représentation mentale de la situation.

Lorsque vient le temps de décrire l'originalité des relations cognitives dans le jeu et en jeu, on retrouve toujours une image ou une représentation qui entretient une relation de dépendance avec les objets de la configuration du jeu. Toutefois, cette dépendance, pour être efficace, doit être ramenée à des objets simples et peu nombreux. Pour les joueurs, l'importance des relations cognitives construites avec leur environnement n'est plus à démontrer. Habituellement, cette relation se construit lors de la confrontation à une configuration du jeu et implique des processus décisionnels de la part des joueurs. Cette relation cognitive non isomorphe au réel doit être aussi rapidement efficace que possible afin d'éviter les tâtonnements tactiques. Cela est confirmé par Edelman (1992) ainsi que Edelman et Mountcastle (1978) qui caractérisent la « dégénérescence » comme toutes les insuffisances, toutes les altérations systématiques qui ne permettent pas une correspondance parfaite entre la forme proposée par un émetteur et la forme assimilée par un récepteur. Alors, cette dégénérescence relève bien des mécanismes de transmission et, dans le jeu, elle ne traduit pas l'altération physique d'un message par le bruit, mais l'altération de la communication elle-même. Dans une situation de jeu, l'importance de la dégénérescence apparaît immédiatement pour la prise de décision et toute diminution de celle-ci est le signe d'une connaissance apprise. Le principe de dégénérescence conduit à affirmer qu'il n'est ni possible ni nécessaire qu'un joueur 


\section{eJRIEPS Hors série n¹ Décembre 2015}

analyse parfaitement toutes les informations liées à la configuration du jeu pour établir une relation satisfaisante avec elle en vue de perturber son équilibre. Cet état d'équilibre étant " apprécié » par le joueur lui-même, il s'introduit bien dans le processus de décision. Du point de vue de l'activité du joueur, il existe donc des éléments du jeu, des relations qui pour une tâche donnée ne présentent pas d'intérêt. Le joueur doit en faire abstraction lorsqu'il résout un problème. Par contre, il existe d'autres relations qui sont indispensables à la résolution de la tâche. A cet effet, Ochanine $(1969,1978)$ distingue les images opératives des images cognitives. Les images cognitives sont le reflet intégral des objets dans toute la diversité de leurs propriétés accessibles. En revanche, les images opératives sont des structures informationnelles spécialisées qui se forment au cours de telle ou telle action en fonction des configurations du jeu. Cette distinction permet à l'auteur de souligner l'ambivalence fonctionnelle de l'image : l'image est, en même temps un instrument de connaissance et un régulateur de l'action. L'image cognitive remplit la fonction d'instrument de connaissance. L'aspect cognitif tend à collectionner le plus d'informations possibles sur la configuration, mais est contradictoire avec une action rapide, alors que l'aspect opératif (rôle régulateur) ne reflète que les aspects utiles des configurations.

Ochanine (1969) définit trois caractéristiques de l'image opérative : (1) la finalisation, (2) le laconisme, (3) les déformations fonctionnelles. La finalisation est la propriété principale de l'image opérative. En effet, on distingue l'image opérative de l'image cognitive du fait que la première se forme au cours d'une action donnée sur une configuration, alors que la seconde est le reflet intégral de la configuration. Par ailleurs, nous avons vu qu'une image opérative peut ne plus être adéquate pour la tâche si au cours de l'activité le but est modifié. Le fait que le joueur doive continuellement ajuster son image opérative aux objectifs poursuivis est une preuve de la finalité de cette construction mentale. De plus, Ochanine (ibid) souligne que la structure opérative de la configuration sera celle qui contiendra le plus petit nombre possible de relations, en nombre suffisant pour la réalisation de la tâche. En d'autres termes, l'image opérative est celle qui, par le plus petit nombre possible de relations, fournira au sujet de l'action le maximum d'informations pertinentes sur la configuration.

La finalisation de l'image opérative a pour conséquence directe la sélection de l'information pertinente. Elle ne retient que ce qui est directement utile à l'action. En ce sens elle est sélective et doit être économique. Nous retrouvons ici l'idée que le reflet subjectif de la configuration construite dans la conscience du joueur n'est pas le reflet d'un 


\section{eJRIEPS Hors série n¹ Décembre 2015}

miroir mais c'est une sélection des informations pertinentes. Ainsi l'image opérative est une déformation fonctionnelle de la réalité. La déformation fonctionnelle est l'accentuation des «points " informatifs les plus importants en fonction de la tâche visée : propriétés de la configuration, ses divers aspects, ses structures partielles Cette déformation est dite fonctionnelle tout d'abord parce qu'elle implique que le reflet soit toujours adéquat à la réalité.

Enfin, on précisera que si le joueur travaille dans un milieu dynamique, sur des configurations dynamiques, le reflet de son activité sera également dynamique. L'aspect dynamique des images opératives correspond, ici, à une anticipation, à une prévision du déroulement des événements qui représente en fait leur reflet anticipé dans la conscience sous forme d'images opératives dynamiques. Ce reflet anticipé n'est pas seulement cognitif. En effet, il permet au joueur de préparer ses actions, mais également de les mettre en œuvre. Le reflet anticipé, combinant les états de la configuration et les réactions adéquates à ces états permet de passer directement de la dynamique de la configuration à la préparation, puis à la réalisation d'actions sur celle-ci. En effet, comme le note Spérandio (1984), l'image opérative n'est pas seulement le reflet subjectif de la configuration, mais aussi le reflet de l'action projetée sur la configuration.

Dans l'évolution de l'image opérative au cours d'expériences successives, les indices significatifs sont exagérés. En d'autres termes, les écarts observés par rapport à la réalité sont des déformations fonctionnelles. Cette étude permet de mettre en évidence que les déformations fonctionnelles, caractéristiques de l'image opérative, n'apparaissent qu'avec l'expérience.

L'image opérative est donc une construction mentale de la configuration du jeu, un reflet dans la conscience du joueur ou du système dans lequel le joueur agit. En outre, elle ne représente que les éléments qui sont pertinents pour le joueur en fonction de sa tâche en cours. Nous pouvons dire que pour agir sur une configuration du jeu le joueur doit donc posséder une image mentale opérative reflétant la structure opérative de la configuration. Cette image permet au joueur d'agir sur la configuration momentanée du jeu qu'elle représente mais aussi de la contrôler s'il s'agit d'un processus en train d'évoluer. Dans ce dernier cas, on peut attribuer une capacité anticipatrice à l'image dynamique opérative. $\mathrm{Ce}$ caractère transitoire suggère que cette image, élaborée d'une part à partir des informations perçues dans l'environnement et, d'autre part, à partir des connaissances en mémoire (configuration prototypique par exemple) soit élaborée dans la conscience $d u$ joueur en relation avec sa mémoire. 


\section{eJRIEPS Hors série n¹ Décembre 2015}

Une étude simple (Gréhaigne, 1986), reprenant, avec ses étudiants, en partie le travail de Mahlo (1969) mais avec de petites séquences vidéo à la place de photographies ou de dessins, a confirmé comment l'image évolue au cours de l'apprentissage d'une activité. Trois groupes de sujets ont été confrontés à l'aide de la vidéo à une configuration du jeu en football. Les joueurs étaient répartis en fonction de leur niveau de connaissances et de pratiques : trois joueurs experts, trois débrouillés et trois débutants. Les joueurs devaient décrire brièvement la configuration du jeu et proposer une réponse pour faire évoluer positivement celle-ci. Les résultats ont mis en évidence qu'en général les détails non marquants sont négligés chez les experts, et que seuls quelques indices significatifs sont décrits. Par contre, les débutants ont réalisé des descriptions très justes de la réalité mais cependant pauvres en ce qui concernent les informations importantes, à savoir les indices pertinents pour répondre à la situation. Quant aux joueurs débrouillés, ils ont exploré partiellement la configuration du jeu avec une centration fréquente sur l'espace lointain et l'avant de l'espace de jeu effectif. On a pu en conclure que l'évolution s'opère d'un joueur débutant encombré par une multitude d'informations, vers un joueur expert qui ne relève que les quelques indices pertinents. Ainsi, dans ce continuum, on peut affirmer qu'une transformation qualitative s'est opérée car même si un expert peut traiter beaucoup d'indices, le qualitatif l'emporte au moment de la décision. Cette expérience a permis de mettre en évidence que les déformations fonctionnelles, caractéristiques de l'image opérative, n'apparaissent qu'avec le temps et l'expérience et, on peut le supposer, quand un certain nombre d'opérations sont traitées en tâche de fond. Ce travail et ce modèle de l'image opérative remettent bien en cause l'équilibre traditionnel décrit entre quantité et qualité des informations traitées par un joueur en vue de prendre une décision. Expérience, déformation, redondance, concision semblent être, ici, les maîtres mots pour qualifier les éléments de la réalité observée.

\section{Configuration du jeu et décision}

Comment le joueur répond-il aux problèmes posés par les exigences des configurations momentanées du jeu ? II doit prendre des décisions: il doit donc réagir par un acte tactique qui est conscient et orienté. Cet acte tactique va tenter de résoudre les problèmes posés par le jeu en faisant des choix utilisant les qualités physiques, les compétences motrices et les connaissances que l'élève possède de l'activité. L'engagement et la motivation jouent aussi un rôle important dans ce processus. 


\section{eJRIEPS Hors série n¹ Décembre 2015}

\section{CONFIGURATIONS DU JEU}

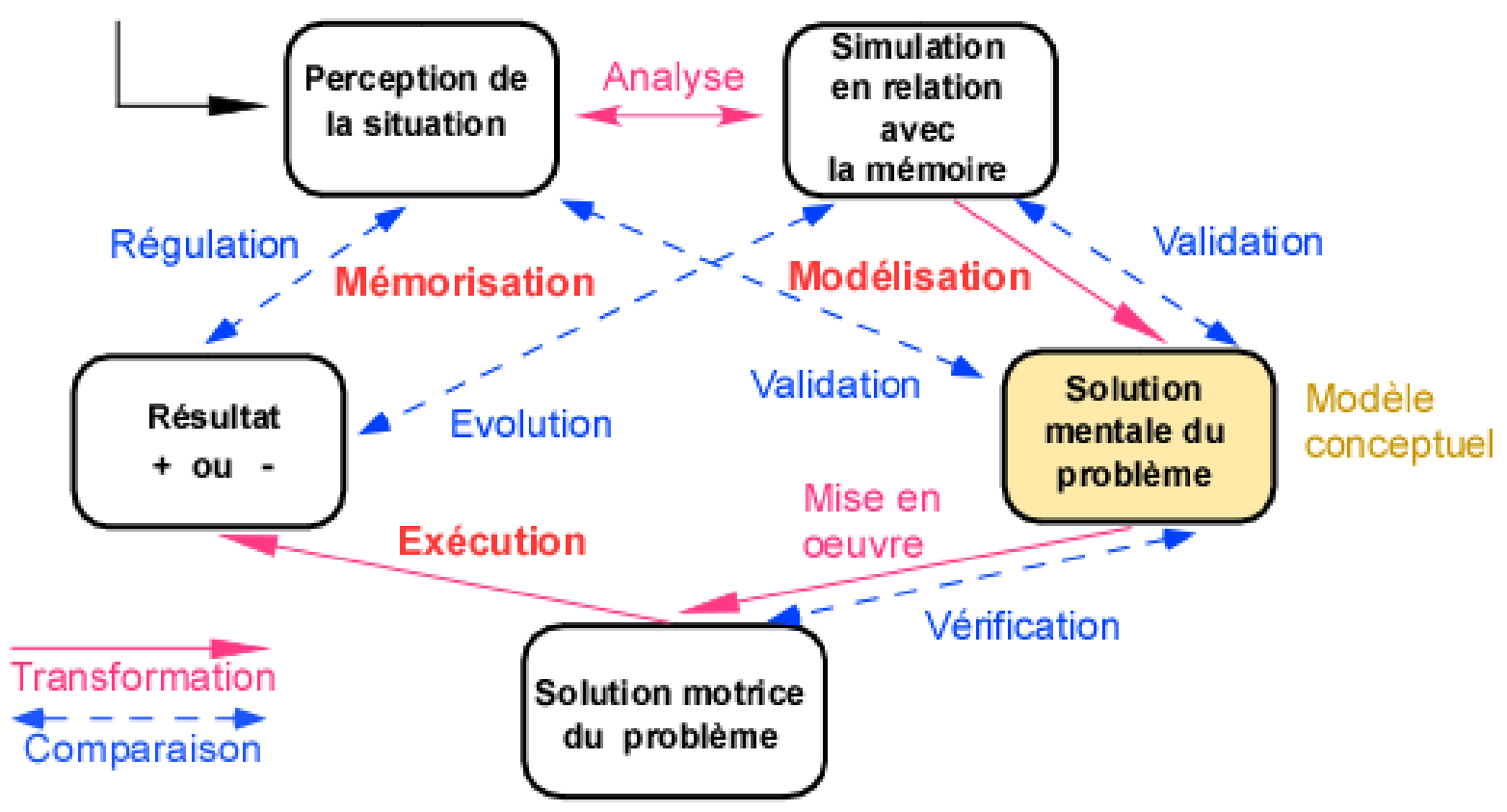

Figure 3. Le modèle de Mahlo (1969) revisité.

Si l'on reprend le modèle bien connu de Malho (1969) nous pouvons, à l'aide de divers processus, préciser les modalités de fonctionnement de cette modélisation. II apparaît bien évident que le modèle conceptuel joue un rôle central dans le développement de la compréhension du système d'opposition (figure 3). La comparaison des données obtenues entre les différents items du modèle entraîne ou non une transformation dans les ressources du joueur.

La perception de la tâche renvoie à la signification et à l'importance que le joueur attribue aux éléments de la situation. Elle consiste à identifier les indices informationnels pertinents par rapport au problème posé. II faut donner ensuite une signification à ces indices. Toute perception signifiante est une perception éducative car elle laisse des traces dans la mémoire qui pourront être réutilisées. Parfois cette signification peut être commune à plusieurs sports collectifs. Plus on augmente son vécu tactique, plus on acquiert de signifiants tactiques. Aussi, le modèle conceptuel permet d'obtenir dans un temps réduit la réponse adéquate au problème posé. Mais il est clair que si le modèle conceptuel n'est pas bien conçu, l'exécution de la solution motrice ne pourra pas être mise en œuvre correctement et provoquer des erreurs. Alors, dans un premier temps d'apprentissage, on met l'élève dans des situations tactiques habituelles. Quand il perçoit bien la situation, on la complexifie. II est essentiel que chaque élève prenne conscience du degré de complexité des situations mises en place. C'est à ce niveau que l'on met en 


\section{eJRIEPS Hors série n¹ Décembre 2015}

évidence la pensée tactique individuelle du joueur et c'est le degré de conscience de la difficulté rencontrée qui va permettre de la résoudre ou pas.

La solution motrice du problème et sa mise en œuvre ne sont pas centrales dans ce modèle mais constituent un maillon de la chaîne de décisions. Les compétences motrices reposent en partie sur des automatismes cognitifs. Ce sont des actions souvent élémentaires ou des successions de gestes simples en réponse à un problème, en partie automatisées à force de travail et d'entraînement. Elles sont caractérisées par la stabilité, la précision et la rapidité du déroulement, aussi ces comportements systématiques ne nécessitent pas une qualité attentionnelle particulière car elles fonctionnent en tâche de fond. Les joueurs sont dans des processus adaptatifs répétés et parfois semblables, mais non identiques en tout point surtout dans la réalisation motrice. Les actions motrices vont représenter des solutions simples au problème posé mais qui ne sont pas automatisées. Chaque situation de jeu impose une adaptation permanente du comportement moteur. Ainsi, plus le joueur rencontre de situations problèmes, plus il va acquérir des réponses adaptées stockées en mémoire. D'où l'importance de proposer des situations variées et complexes aux joueurs afin qu'ils développent leur compréhension du jeu. Parfois la configuration du jeu n'a pas de solution dans la mémoire du sujet et c'est l'invention par le joueur d'une action innovante qui lui permet d'adapter son comportement par rapport au problème posé. Cette action innovante constitue une réponse nouvelle qui sera à stabiliser (si elle s'avère de nouveau utile) afin de pouvoir l'utiliser ultérieurement.

\section{Quelques circulations typiques du ballon}

Nous avons montré (Gréhaigne, Caty, \& Marle, 2004) la nécessité d'étudier le passage d'une configuration de jeu à une autre configuration de jeu pour mieux comprendre l'évolution d'une séquence de jeu. Au cours de l'action, les configurations momentanées du jeu évoluent d'un état à un autre et ainsi de suite mais parfois la même forme de configuration réapparaît: un prototype en quelque sorte au sens de ce qui est en adéquation avec un modèle donné. Mais, c'est vrai, il n'y a pas de consensus sur ce qui constitue un « prototype ». Le mot est également souvent utilisé de façon interchangeable avec le mot «modèle» ou signifie une ébauche fonctionnelle d'un objet. Dans les sports collectifs, en testant différentes configurations momentanées du jeu, on vise, en premier au travers de prototypes, à découvrir le fonctionnement de la structure du jeu. Dans l'équipe, ces modèles sont destinés à aider à la prise de décision interne et constituent une partie du fond de jeu qui permet la compréhension par chacun de l'évolution des 


\section{eJRIEPS Hors série n¹ Décembre 2015}

rapports d'opposition. L'expérience qui consiste souvent à confronter un ou des joueurs à un prototype est principalement utilisée pour comprendre les décisions de ceux-ci. Aussi, ce type de modèle peut permettre une évaluation précoce de la façon dont un joueur interagit avec les éléments du jeu, en particulier les partenaires en regard des différentes actions dans un rapport de forces donné. Finalement, nous avons dénommé ce type de configurations "prototypiques » au sens où il représente un modèle original, archétype d'un modèle qui se reproduit (Gréhaigne, 2007). L'étude de l'ensemble des configurations prototypiques devrait permettre aux élèves de construire des prototypes (cf. images opératives, Ochanine, 1978) par catégorisation de formes géométriques, de classes de propriétés d'objets et enfin des catégorisations de relations temporelles en vue d'être plus efficaces en jeu. On vise, dans ce cas, la mise à jour d'invariants pour reconnaître les configurations momentanées du jeu et décider vite à propos de la suite à donner au jeu (Gréhaigne, ibid). Ici, deux principes cognitifs semblent être concernés. Le premier touche la fonction de catégorisation qui est de fournir des informations pertinentes avec peu d'effort. Le second concerne la structure de l'information fournie qui postule que les configurations du jeu sont perçues sous forme d'informations structurées. En effet, entre ces configurations il n'y a pas forcément des points communs, mais sûrement des similitudes et des relations, parfois nombreuses. Néanmoins, une question fondamentale reste posée : le prototype constitue-t-il une image mentale partagée entre les joueurs, à partir de laquelle ils peuvent déduire les propriétés prototypiques, ou plutôt un faisceau de traits typiques, à partir duquel ils pourront juger du degré de similarité de telle ou telle configuration ? Dans ce dernier cas, une réflexion reposant sur l'analogie (Gréhaigne, Godbout, \& Zerai, 2014 ; Zerai, Gréhaigne, \& Godbout, 2013) peut aider les joueurs à décider. Pour eux, le résultat final est constitué par une série de modèles de configurations avec une compréhension de leurs forces et de leurs faiblesses. On peut souligner maintenant le rôle important d'indices dits " de surface ", comme dans l'image opérative à l'occasion de la récupération en mémoire d'une configuration analogue. Auparavant, on avait tendance à ne se focaliser que sur la structure du problème analogue. Le joueur devra maintenant apprendre à centrer son attention sur le jeu en train de se dérouler et sur les indices, tel qu'il s'y trouve engagé afin d'en déceler la cohérence et profiler l'évolution probable qu'il produit. En un mot il doit s'appuyer sur le « potentiel de la situation » (Jullien, 1996). Deux notions se trouvent ainsi au cœur de l'analyse du jeu : d'une part, celle de configuration du jeu telle qu'elle s'actualise et prend forme dans le rapport de forces en cours et, d'autre part, celle de potentiel d'une telle situation dans 


\section{eJRIEPS Hors série n¹ Décembre 2015}

laquelle le joueur se trouve impliqué et dont il doit tirer parti. Cette option consiste à s'attacher à suivre le cours de choses, à ne pas forcer le réel à partir d'un modèle soidisant parfait et à ne pas interrompre le flux des informations et des indices par une analyse trop rationnelle voire simpliste. Ici, résister à ce flux semble vain puisque c'est s'attaquer à des actualisations particulières et mouvantes du rapport des forces. Cette analyse confirme le bien fondé de notre position en regard de la technique; les compétences motrices seules sont incapables de résoudre les problèmes posés par le jeu et l'on est bien dans un système décisionnel complexe qui ne saurait se ramener à du contrôle ou de l'apprentissage moteur de façon exclusive. Un joueur stratège n'est pas seulement un technicien, c'est un joueur qui décide à partir du réel en l'accompagnant, en repérant ce qui est en train d'émerger pour vaincre la résistance de l'adversaire.

Mais, pour en revenir précisément à la relation entre les compétences motrices et les configurations du jeu, quelques étapes bien caractéristiques en relation avec des postures de lancer semblent jalonner ce processus. A cette occasion nous complétons des éléments exposés dans le livre "l'intelligence tactique " et plus spécialement dans le chapitre « développer la pensée tactique à l'école » (Gréhaigne \& Caty, 2014).

4. 1. Les débutants

II est possible d'observer que les joueurs débutants, face à une défense en barrage, subissent la situation plus qu'ils ne la dominent.

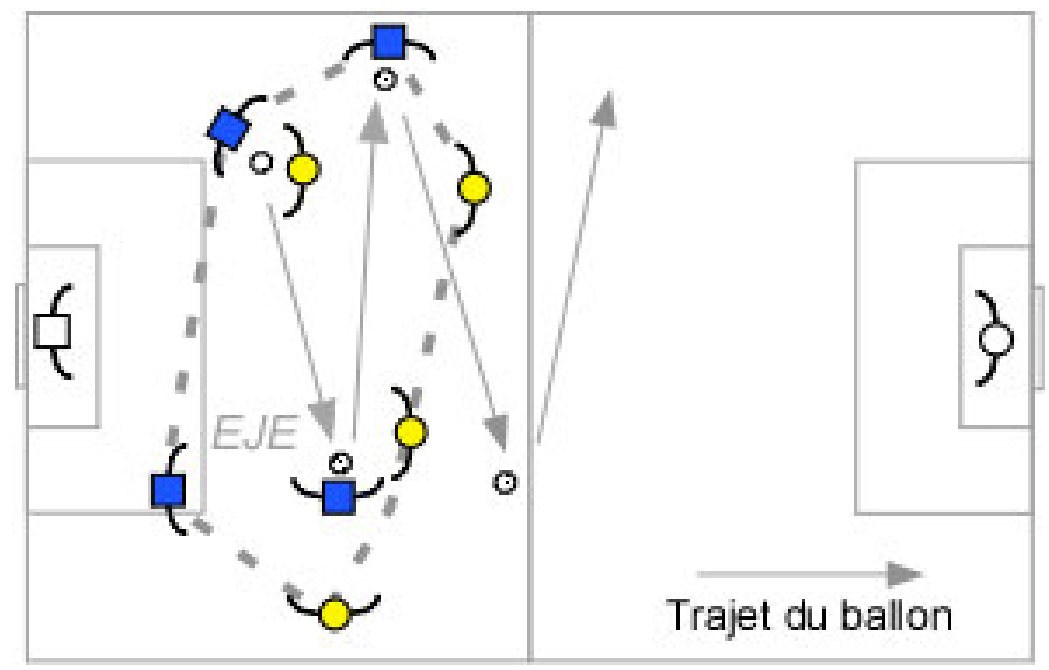

Figure 4a. Une circulation de la balle à plat jusqu'à la perte du ballon.

La figure $4 a$ illustre une circulation de la balle caractéristique des débutants quand le lancer long n'est pas encore bien construit. Après une mise en jeu sur la ligne de but, les attaquants s'échangent le ballon à l'aide de passes courtes, en cloche et en travers car la 


\section{eJRIEPS Hors série n¹ Décembre 2015}

défense, qui occupe l'axe central ou le devant du porteur de balle, les empêche de passer le ballon en avant. Cette circulation de la balle à plat, dans la dimension forte de la défense entraîne très fréquemment une perte du ballon au profit de l'équipe adverse et se traduit très souvent par un tir spontané du récupérateur. La motricité habituelle (courir, sauter, lancer) sert de soubassement pour les compétences motrices.

Pour dépasser ce niveau débutant, il faut aider à la construction d'un lancer assuré, assez tendu et destiné à un coéquipier en relation avec la prise en compte des informations qui permettent la gestion et l'exécution de l'échange de balle. Cette action est alors possible si l'élève est capable de concevoir le réceptionneur comme continuateur de sa propre action. Néanmoins, bien souvent, la circulation de la balle et le déplacement des joueurs s'organisent dans l'axe central (figure 4b), entre les différents arrêts du joueur en possession du ballon. Lors de ces arrêts, l'espace de jeu effectif (EJE) se reconstitue en compression devant le porteur de balle constituant un barrage difficile à dépasser. Pour résoudre ce problème et si le joueur en possession du ballon s'en sent capable, on peut voir aussi apparaître chez celui-ci l'utilisation du dribble en contournement de l'espace de jeu effectif, pour passer la balle ou tirer au but. D'une manière générale chez les débutants, l'EJo avance ou recule au gré des pertes, des récupérations du ballon ou des affrontements mais dans l'axe du terrain but attaqué / but défendu.

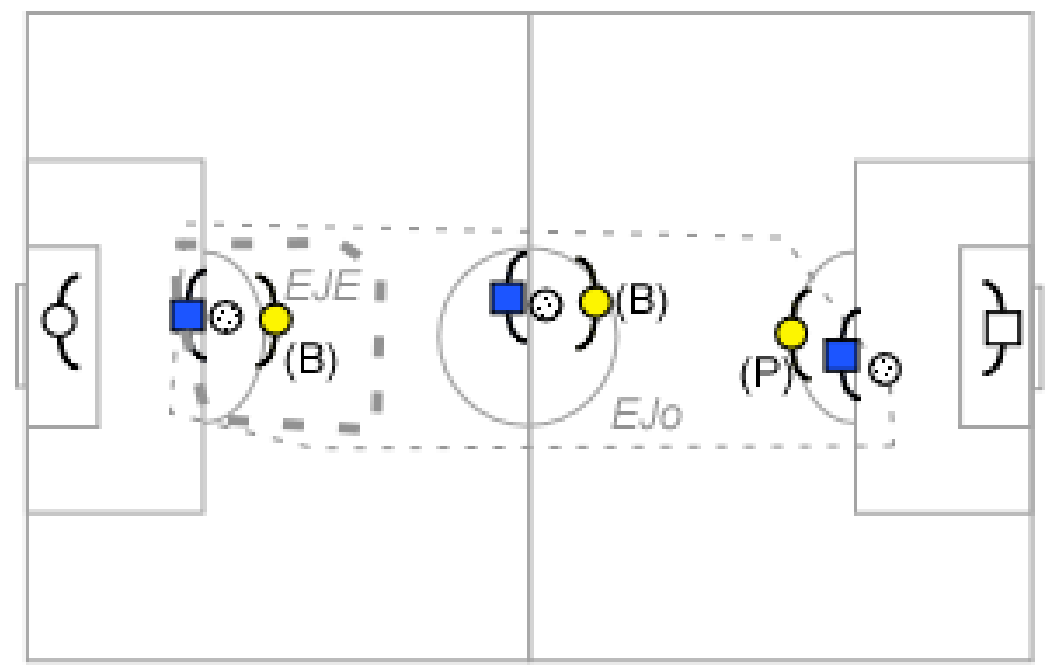

Figure 4b. Emplacements d'affrontements dans l'axe but attaqué / but défendu où le ballon circule.

Un des problèmes récurrents posés par les outils d'analyse et d'observation proposés en sport collectif aux débutants est constitué par le fait que les élèves doivent constamment 


\section{eJRIEPS Hors série n¹ Décembre 2015}

effectuer une rotation cognitive du plan "vue d'avion" pour les explications à une vision dans le plan horizontal et en profondeur pour la réalité du jeu. Ce n'est pas sans poser des questions de transposition et de compréhension car, dans le plan horizontal, cela revient à traiter des effets de largeur, de profondeur et de distance qui sont très différents d'une vue de dessus.

4. 2. Récupération au milieu du terrain et balle vers l'avant Après la construction de la passe en déplacement et de sa réception, les progrès sont liés au degré de compréhension du jeu par les joueurs. La motricité devient plus élaborée avec l'apparition dans les compétences motrices de la différenciation fiable entre pousser et frapper un ballon, et l'évolution vers un lancer plus long. Les points importants à ce niveau de confrontation sont constitués par la construction de l'espace vide en avant du réceptionneur où celui-ci va se trouver dans un instant très proche ainsi que la gestion de la distance porteur de balle / adversaire direct pour permettre l'échange de la balle (figure 5). Cela produit parfois un jeu avec des passes longues hasardeuses qui entraînent de nombreuses pertes de balles et les séquences de jeu n'excèdent que rarement 2 à 3 échanges de balle.

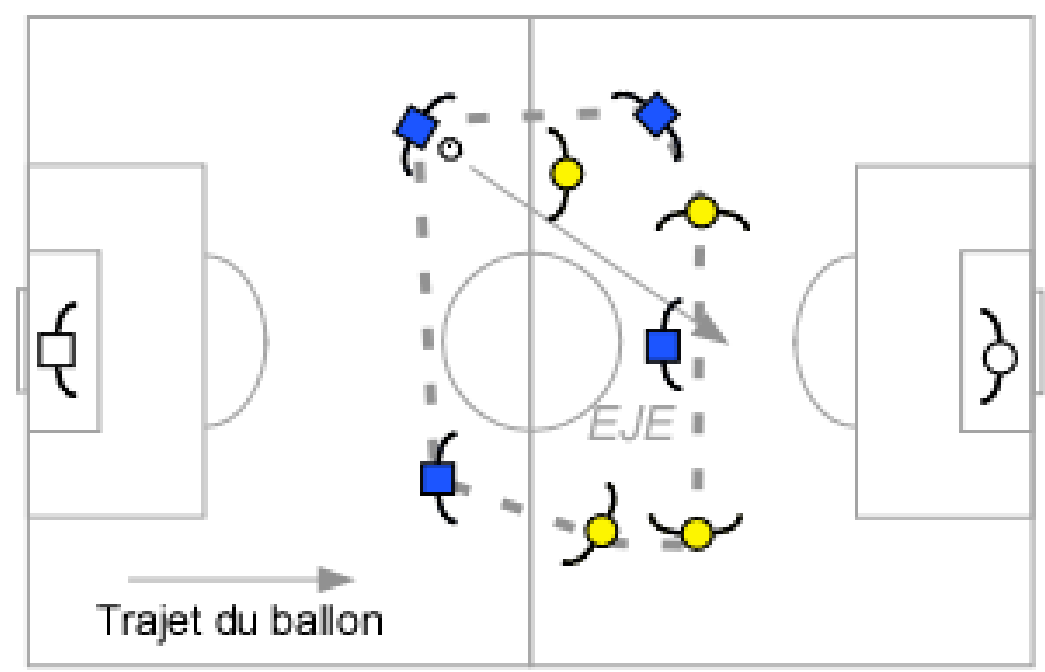

Figure 5. Passe en avant du joueur qui court

Des espaces de jeu effectifs en expansion (Gréhaigne, 2009) apparaissent de plus en plus souvent avec, en particulier, une succession de contre-attaques. Ensuite, les compétences motrices s'affinent en particulier avec la possibilité de jouer en mouvement et de plus en plus vite. Par contre, ce style (de balle qui fuit en avant est difficile à attraper et produit de nouvelles transformations de la motricité pour capturer la balle. Lors de la 


\section{eJRIEPS Hors série n¹ Décembre 2015}

transition entre ces deux modélisations (balle vers l'avant et relais), l'EJo augmente progressivement, la continuité des échanges étant mieux assurée.

4. 3. Montée de balle en échanges de balle

Un meilleur enchaînement dans les échanges de balle et des progrès dans la réception conduisent à des séquences de jeu plus longues avec un nombre de passes qui augmente (figure 6).

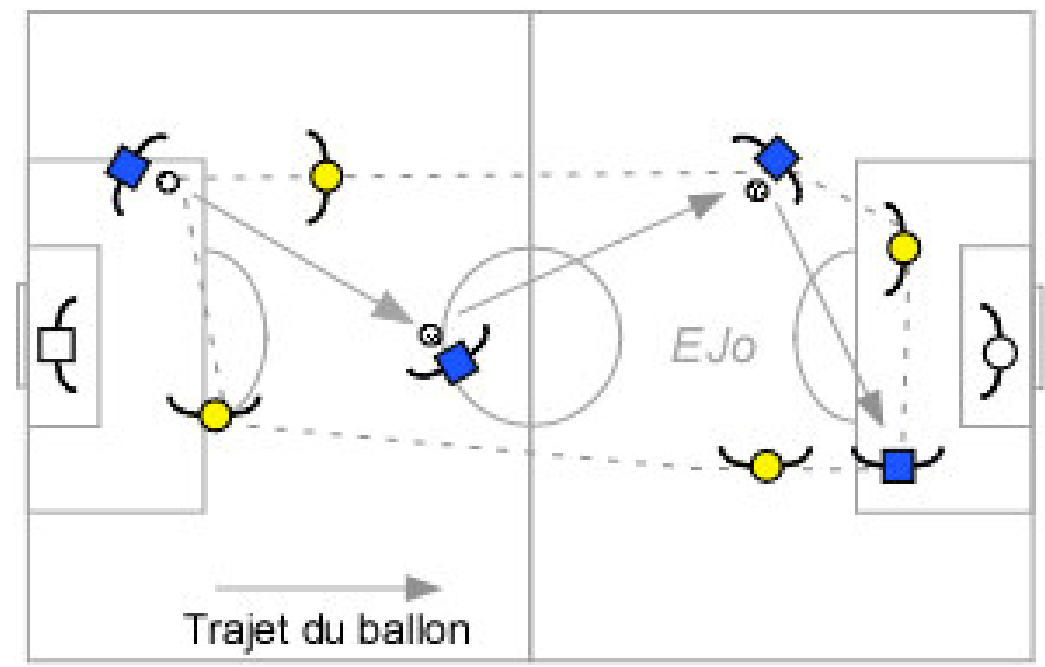

Figure 6. Montée de balle en relais et espace de jeu occupé.

L'espace de jeu occupé (EJo) s'agrandit toujours vers l'avant. Un jeu en relais amène assez souvent la balle dans la zone de marque ce qui permet de tirer au but plus souvent. L'amélioration de la circulation du ballon produit parfois une accélération du jeu qui est propice à une prise d'avance de l'attaque sur le replacement défensif.

4. 4. Contre-attaque ou attaque de position

Ici, les compétences motrices stabilisées sont au service d'un projet tactique qui vise souvent à alterner les circulations de la balle et celles des joueurs en fonction du jeu de l'adversaire. Selon les caractéristiques de l'opposition, les joueurs utilisent soit la contreattaque soit l'attaque rapide (figure 7), le plus souvent avec une passe longue au départ. 


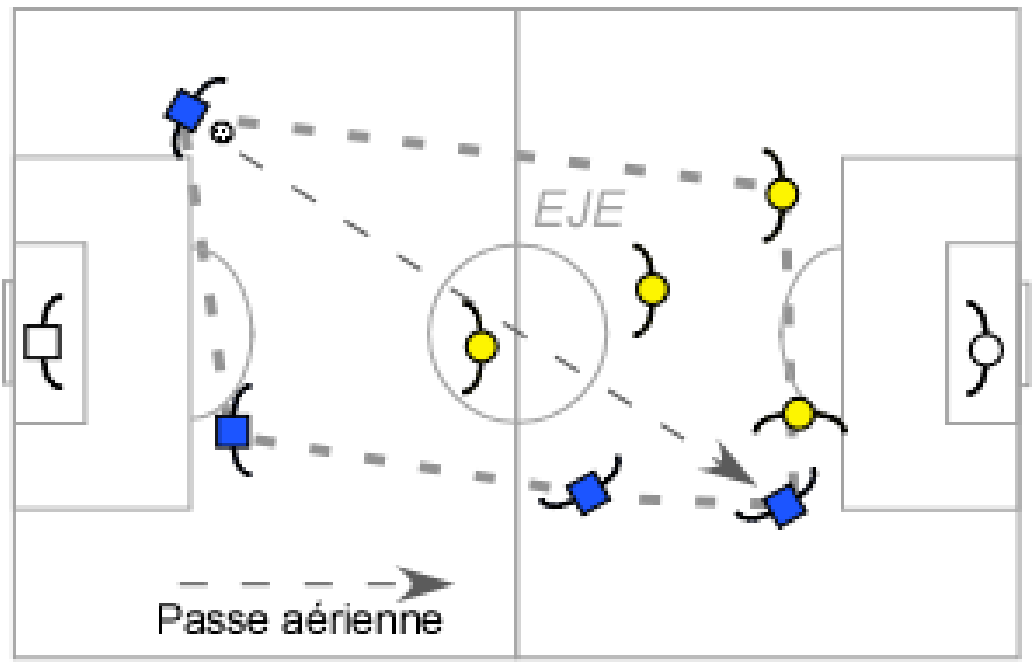

Figure 7. Le porteur de balle effectue une passe longue, aérienne vers l'avant de l'EJE

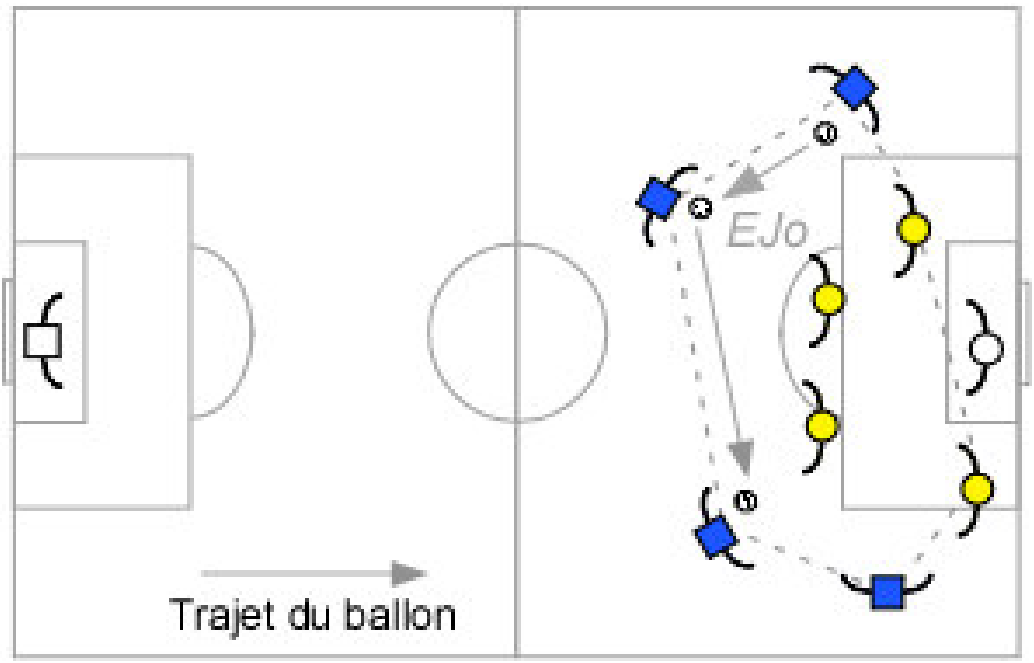

Figure 8. Attaque de position face à une défense de zone

Par contre, si les adversaires se replient vite les attaquants sont face à une défense en barrage et doivent avoir recours à une attaque de position (figure 8). Ce dernier cas propose une situation plus difficile pour l'attaque avec un espace de jeu occupé relativement stable devant le but. Une des solutions consiste à tirer de loin, compétence motrice qui n'est pas toujours construite par tous et qui n'est pas toujours judicieuse dans la mesure où sa réussite est conditionnée entre autre par de nombreux facteurs (vitesse et distance de frappe, angle de frappe, qualité du gardien, etc.). Néanmoins, c'est sans doute le bon moment pour l'enseigner pour les attaquants ou les défenseurs qui peuvent, ainsi, éloigner le ballon de la zone de but.

Ici, la tactique individuelle ne peut bien se modéliser qu'en concevant une évolution du jeu discontinue dans le temps. Cet ensemble est bien sûr à mettre en relation avec le rapport 
eJRIEPS Hors série n¹ Décembre 2015

d'opposition momentané et les compétences motrices des joueurs pour obtenir une réponse adaptée.

\section{Les configurations prototypiques}

A l'aide de ces éléments, nous allons illustrer dans les figures qui suivent les configurations prototypiques les plus habituelles que nous avons identifiées (Caty \& Gréhaigne, 2005) ; Caty, Meunier, \& Gréhaigne, 2007). Cet ensemble devrait permettre aux élèves de construire des prototypes par catégorisation de formes géométriques, de classes de propriétés d'objets et enfin des catégorisations de relations temporelles en vue d'être plus efficaces en jeu. On vise ici la mise à jour d'invariants pour reconnaître et décider vite à propos des configurations momentanées du jeu. L'origine et les circonstances qui ont présidé à la récupération du ballon constituent des aspects critiques pour notre analyse et le développement de la pensée tactique chez les élèves. C'est pourquoi, attirer l'attention des joueurs sur des points clés peut servir avant l'action dans la façon de comprendre, d'organiser, de préparer, et de répondre rapidement au besoin du jeu ou du geste. Ainsi des modèles peuvent être visualisés avec des manipulations d'images susceptibles de faire réfléchir les sujets sur comment faire. En un mot, comprendre pour réussir. Dans les figures qui suivent les positions des joueurs et la place de l'espace de jeu effectif (EJE) sont présentées avec le support de l'activité football mais pourrait l'être aussi bien en basket-ball ou en football (handball).

5. 1. Les emplacements de l'affrontement

La figure $9 \mathrm{a}$ illustre un espace de jeu effectif en zone défensive, avec un ballon en arrière de l'EJE dans l'axe but attaqué / but défendu avec une défense en barrage (ce type d'affrontement pouvant se situer, également, à la périphérie arrière droite ou gauche). Le danger le plus immédiat est constitué par une perte de balle à cet endroit qui permet au défenseur de devenir premier attaquant avec une position de tir très favorable. Sinon, la montée de balle vers une position de tir reste à effectuer.

La figure 9b montre un espace de jeu effectif toujours en zone défensive, avec un ballon en avant de l'espace de jeu effectif (EJE) dans l'axe but attaqué / but défendu avec une défense à la poursuite. C'est une situation assez favorable pour l'attaque mais il reste à parcourir une grande partie du terrain avant d'arriver à une bonne position de tir. Le fait de tenter cette action dépend de la confiance que le joueur a en sa vitesse et dans sa conduite de balle et de la distance avec son premier adversaire direct. 
eJRIEPS Hors série n¹ Décembre 2015

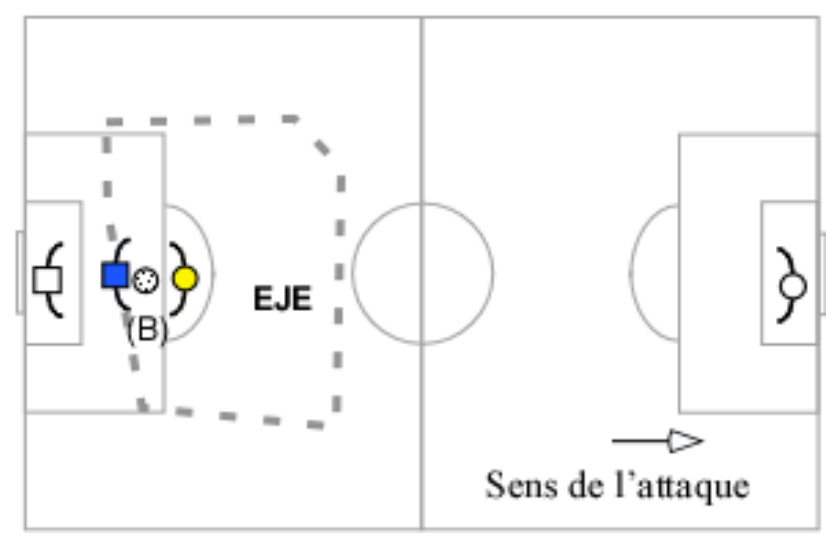

Figure 9a

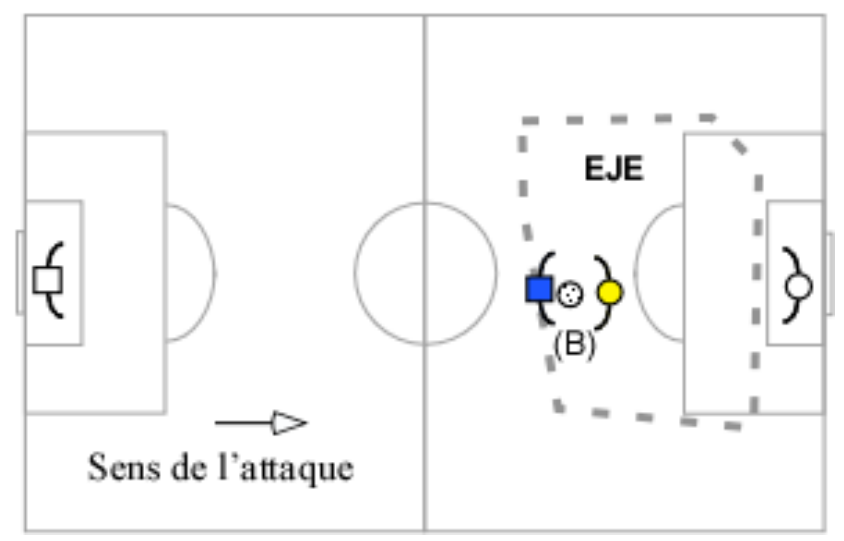

Figure 9c

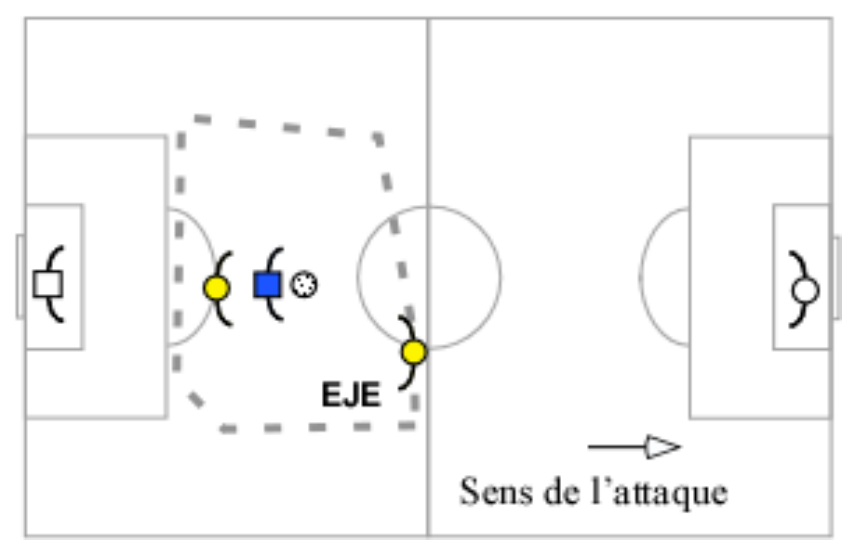

Figure 9e

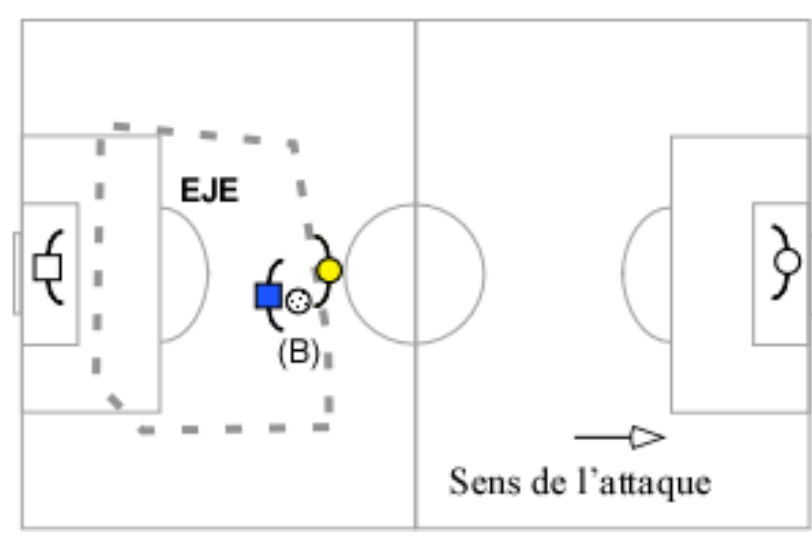

Figure $9 b$

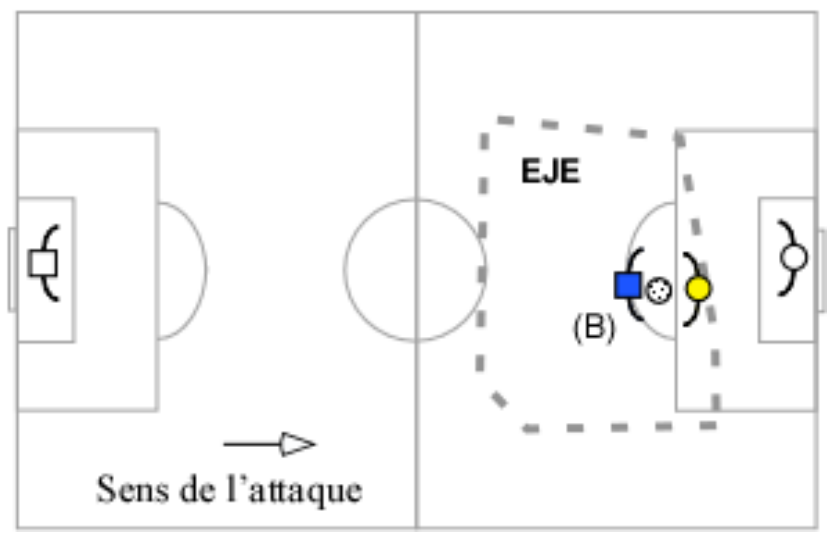

Figure 9d

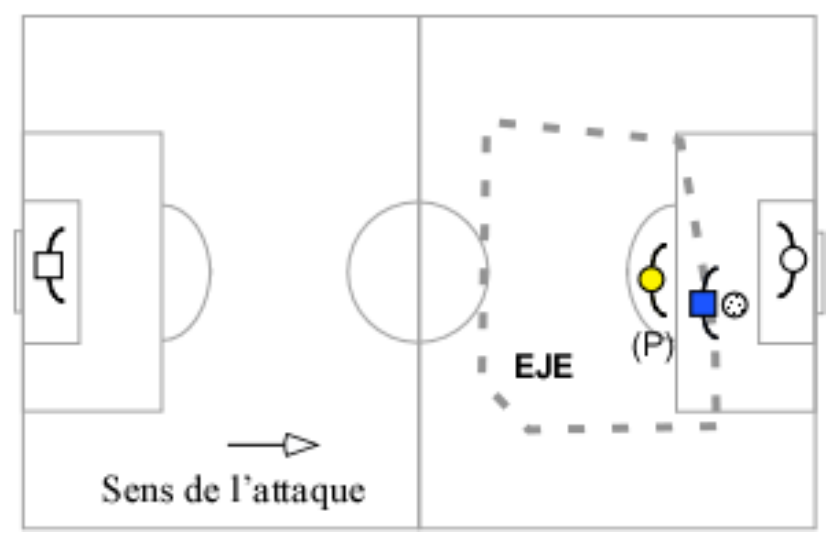

Figure $9 f$

Figure 9. Position de l'EJE sur le terrain et emplacement de l'affrontement avec défense en barrage notée $(B)$ et défense à la poursuite notée $(P)$. 


\section{eJRIEPS Hors série n¹ Décembre 2015}

La figure 9c illustre un espace de jeu effectif en zone offensive, avec un ballon en arrière de l'EJE dans l'axe but attaqué / but défendu avec une défense en barrage. Ce type d'affrontement peut se situer, également, à la périphérie arrière droite ou gauche. Cela renvoie à une attaque de position face avec une défense entre le ballon et la cible. La figure 9d illustre un espace de jeu effectif en zone offensive, avec un ballon à l'avant de I'EJE dans l'axe but attaqué / but défendu avec encore un défenseur en barrage. Ici le porteur de balle doit tenter de passer son adversaire pour s'ouvrir le chemin du but.

La figure 9e distingue un espace de jeu effectif en zone défensive, avec un ballon en milieu de l'EJE dans l'axe but attaqué / but défendu avec une défense en partie en barrage en partie à la poursuite. Ici la localisation du dernier défenseur est déterminante pour la suite du jeu. La figure 9f illustre un espace de jeu effectif en zone offensive, avec un ballon à l'avant de l'EJE dans l'axe but attaqué / but défendu. Ici le porteur de balle doit tirer rapidement au but (la défense étant à la poursuite).

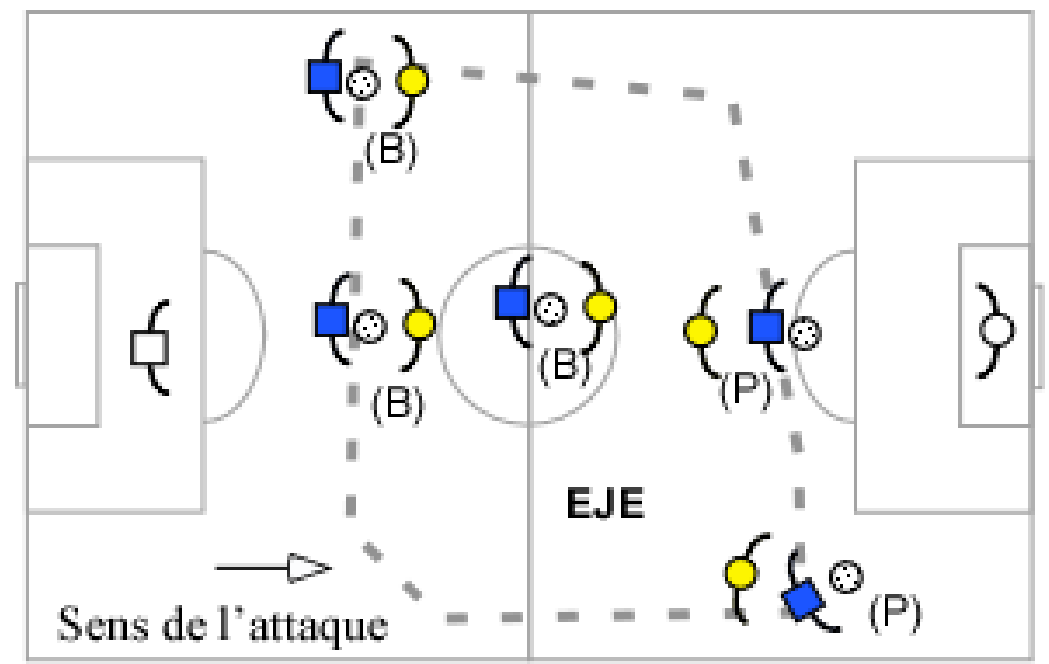

Figure 10. Récapitulatif des emplacements les plus courants dans l'espace de jeu effectif.

La figure 10 récapitule les emplacements que peut occuper un affrontement $1 \times 1$ avec défense soit en barrage soit à la poursuite dans l'axe central ou la périphérie de l'aire de jeu. Les ballons à la périphérie devront être quand même ramenés dans une position proche de l'axe du terrain pour espérer une tentative de réalisation aisée.

5. 2. Les modes de circulation

La figure 11a montre le cas typique d'une contre-attaque sur dégagement du gardien de but en handball ou en football. Dans le cas du dégagement du gardien de but, celui-ci envoie la balle sur un joueur placé en avant qui a anticipé ou qui n'est pas revenu en défense. 


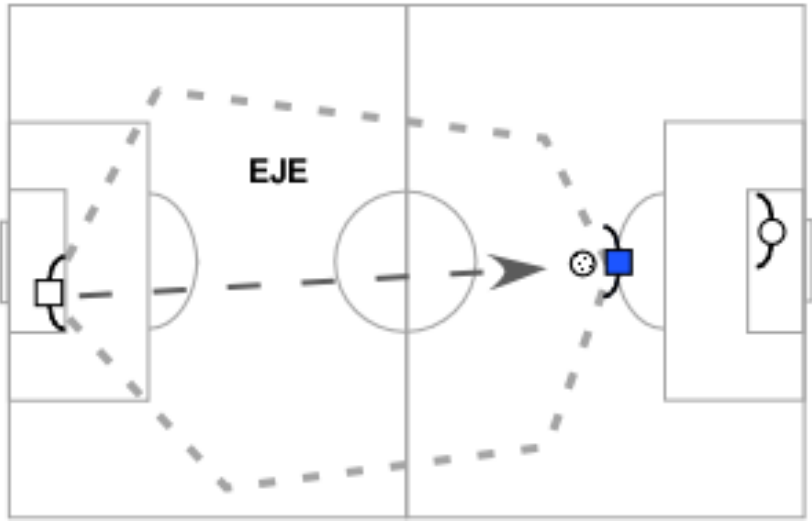

Figure 11a. l'EJE et types de contre-attaque

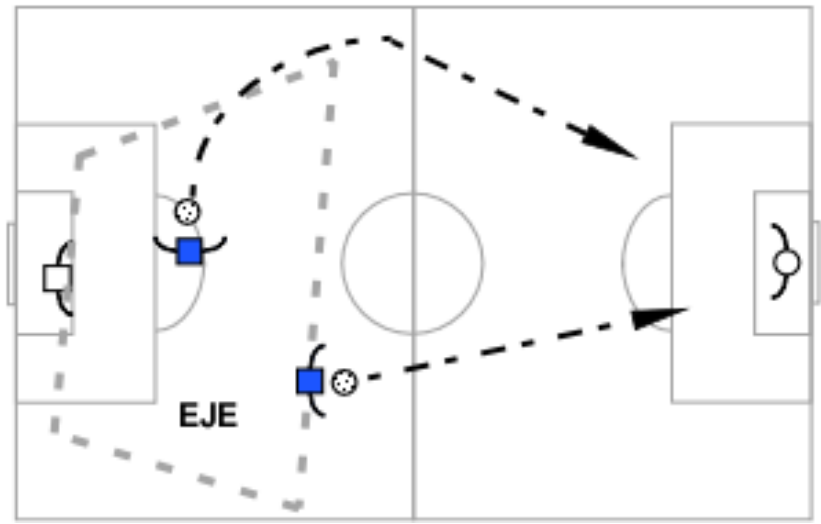

Figure 11b. l'EJE et types de contre-attaque

La figure $11 \mathrm{~b}$ distingue deux cas. Pour le premier, c'est la situation favorable pour l'attaque où le porteur de balle est en avant de l'EJE et n'a pas d'adversaire direct près de lui. Cette configuration est proche de celle décrite dans la figure $9 \mathrm{~b}$ mais en partant de la périphérie. Dans le deuxième cas, une défense encore en partie en barrage oblige le porteur de balle à une stratégie de contournement avec une course curviligne. Si le joueur qui récupère le ballon ne se sent pas capable d'aller seul à la cible, il s'arrête et passe le ballon, ce qui donne du temps à la défense pour se replacer. En football, le joueur s'installe sur le mode "je frappe dans la balle" et "je cours derrière" avec les dangers de perte de celle-ci qui ne reste pas souvent à distance de jeu.

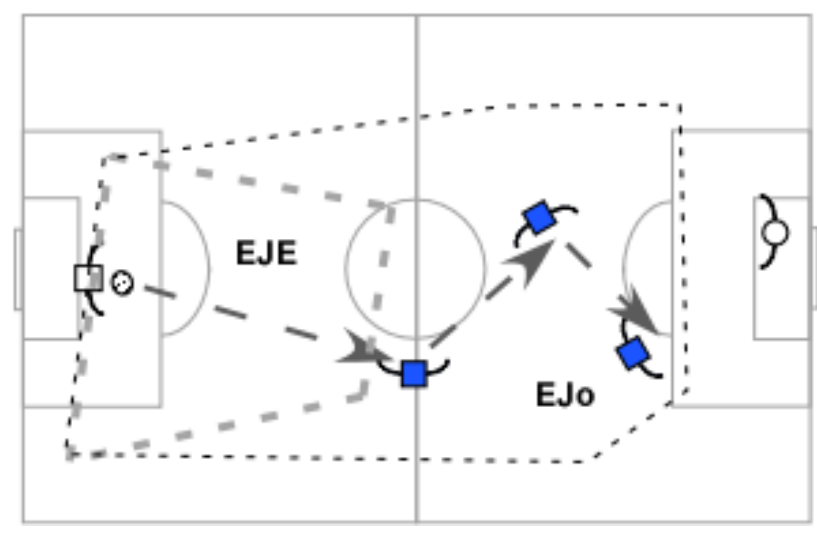

Figure 12a. l'EJE et types de contreattaque

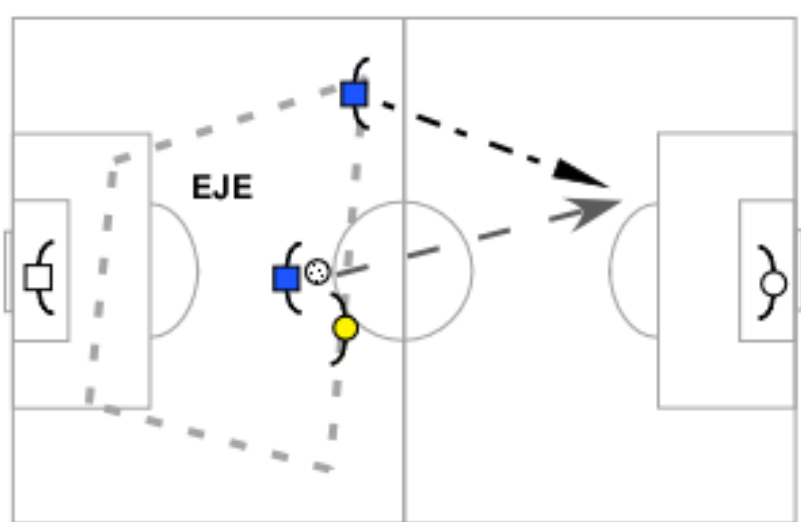

Figure 12b. l'EJE et types de contreattaque

La figure 12a propose une configuration de jeu initiale où l'espace de jeu effectif est également stabilisé dans le demi-terrain défensif. Sans doute suite à une récupération basse à l'arrière de l'espace de jeu effectif, l'équipe qui vient d'entrer en possession du 


\section{eJRIEPS Hors série n¹ Décembre 2015}

ballon met en place un jeu en échanges de balles rapides avec peu de passes qui conduit le plus souvent à un tir. En football ce type de configuration donne un jeu en déviation, le plus souvent à une touche de balle (voir sur ce sujet les travaux de Dugrand, 1989 ou de Lemoine, 2003).

La figure $12 b$ illustre une configuration initiale de jeu quand l'espace de jeu effectif s'est également stabilisé dans le demi-terrain défensif. C'est une récupération à l'avant de l'espace de jeu effectif suivie d'une passe longue en direction d'un partenaire qui a fait un appel de balle. Ici, le passeur lance la balle dans un espace libre où le receveur sera dans quelques instants.

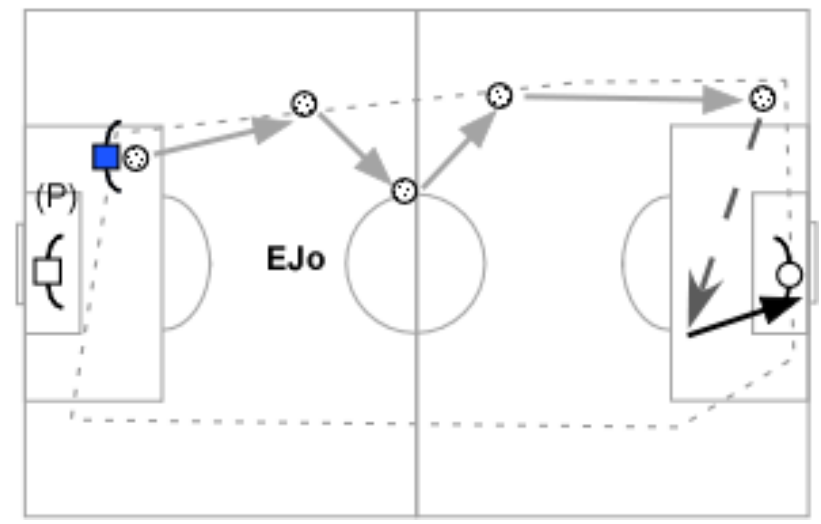

Figure 13a. Conduite et la circulation de la balle

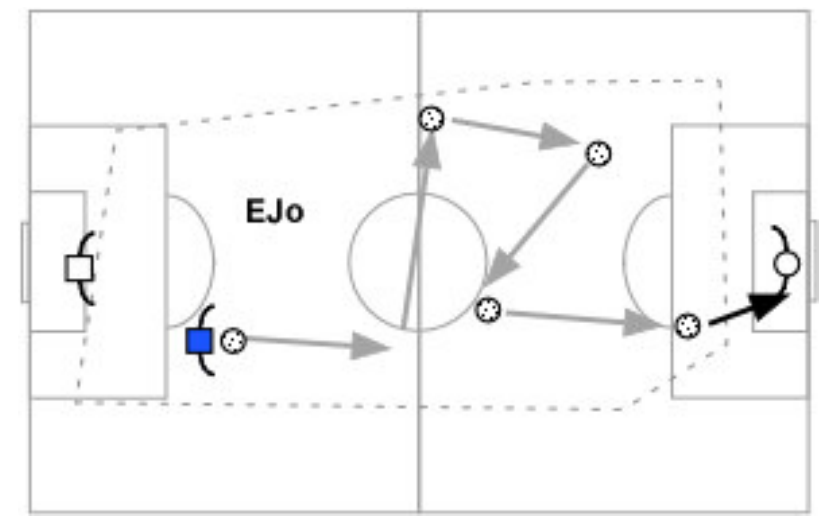

Figure 13b. Conduite et la circulation de la balle

La figure 13a illustre aussi une configuration de jeu initiale où l'espace de jeu effectif s'est également stabilisé dans le demi-terrain défensif. Après récupération du ballon à l'arrière de l'EJE, il s'ensuit un jeu de transition avec de courtes passes et un centre suivi d'un tir au but. La figure 13b distingue une circulation de la balle avec une montée lente pour amener le ballon en avant de l'EJE et tirer au but. Une caractéristique dans ces types de jeu «balle en avant » est constituée par le fait qu'à un certain moment de la montée de balle, «le ballon recule ». Cette circulation temporaire du ballon de l'avant vers l'arrière permet parfois de créer un décalage temporel. L'attaque prend donc paradoxalement de l'avance sur le replacement défensif. Ainsi, la séquence de jeu devient un peu plus longue, même si, en jeu réduit, elle dépasse rarement quatre ou cinq échanges de balle. Les figures $14 \mathrm{a}$ et $14 \mathrm{~b}$ proposent un espace de jeu effectif qui reste devant le but adverse. Ce fait constitue souvent l'illustration d'une défense de zone qui a choisi de s'adosser à sa 


\section{eJRIEPS Hors série n¹ Décembre 2015}

ligne de but ou d'une difficulté de l'équipe en possession du ballon pour monter le ballon et ainsi s'éloigner de son propre but.

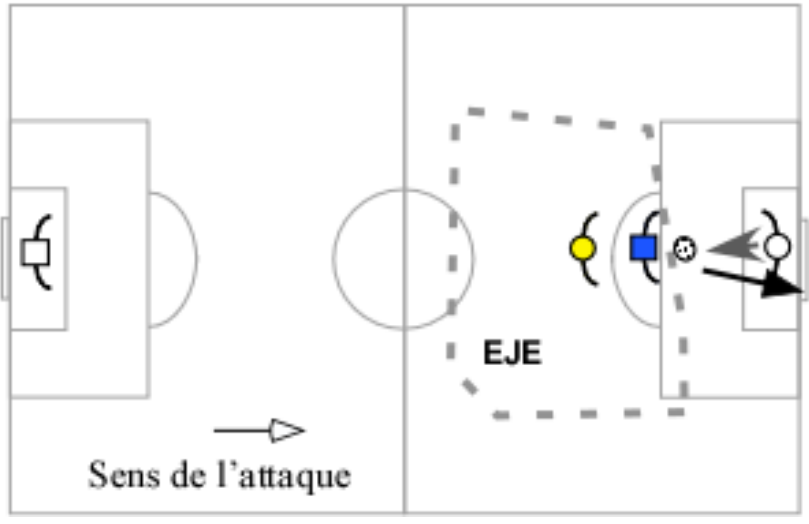

Figure 14a. Récupération sur mauvais

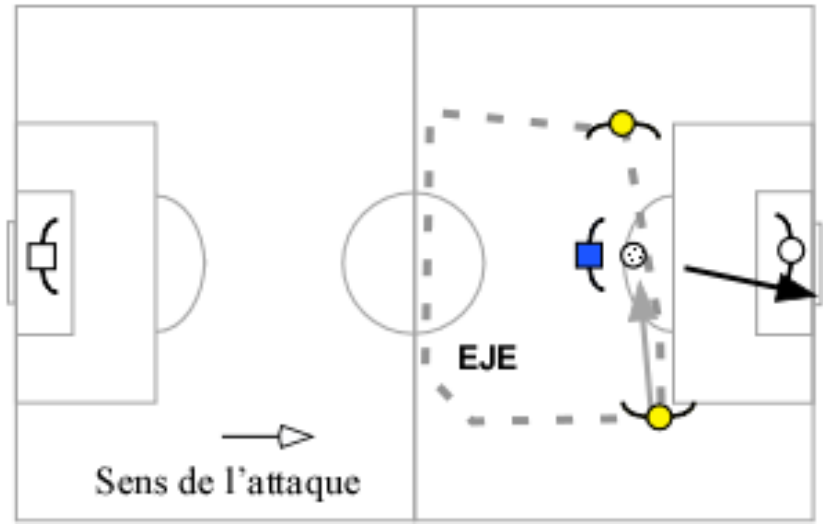

Figure 14b. Interception et tir immédiat renvoi et tir

La figure 14a présente une configuration des plus courantes chez les débutants avec une récupération haute à l'avant de l'espace de jeu effectif suite à un mauvais dégagement du gardien de but, puis un tir quasiment immédiat. La figure 14b illustre une variante de ce type de configuration où une circulation de la balle à l'arrière de l'EJE est interceptée par un adversaire qui rodait par là. Une autre manière d'obtenir ce type de configuration peut faire suite à une passe haute par-dessus l'espace de jeu effectif. Elle peut apparaître aussi après un corner ou une remise en jeu à la main, voire d'une sortie de but. On la retrouve également avec des joueurs plus évolués en conséquence d'un pressing quand les attaquants s'échangent la balle à l'arrière de l'espace de jeu effectif dans leur propre demi-terrain.

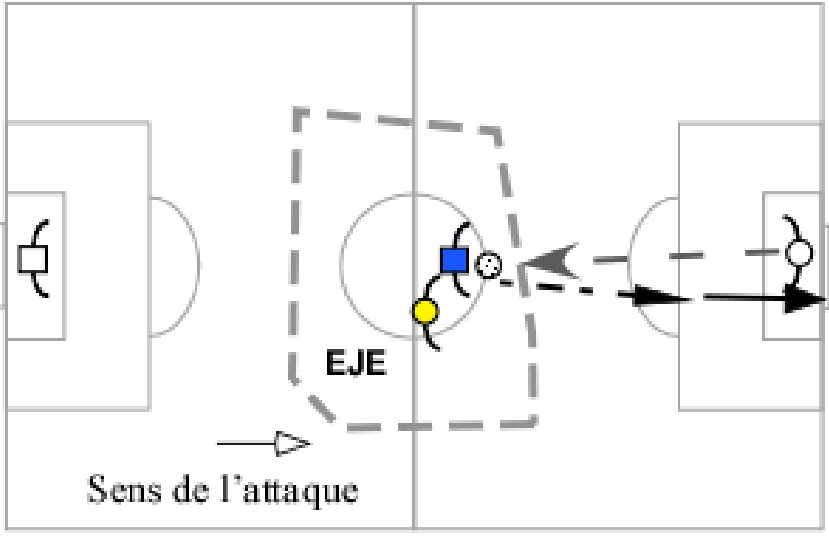

Figure 15a. Récupération au milieu, Figure 15b. Jeu classique au milieu conduite de balle et tir

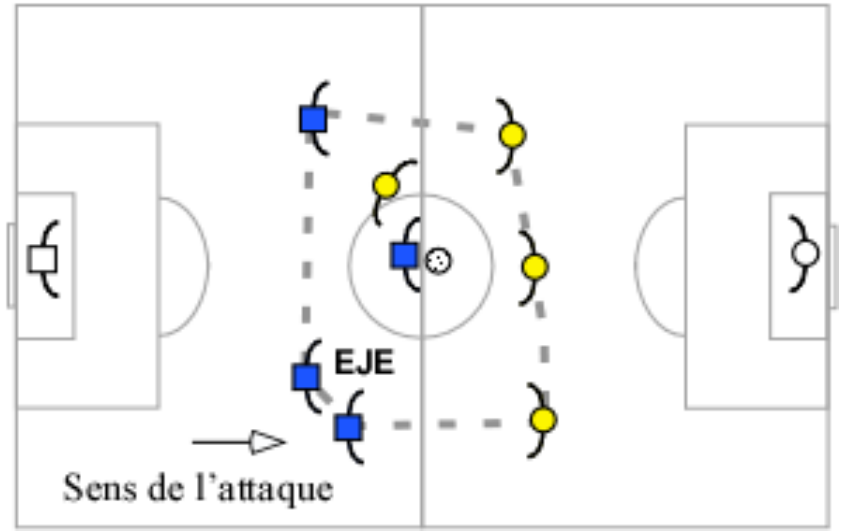




\section{eJRIEPS Hors série n¹ Décembre 2015}

La figure 15a montre avec un espace de jeu effectif au milieu du terrain et un porteur de balle au milieu de l'EJE qui décide, à la suite à un mauvais dégagement du gardien ou une récupération active de la balle, d'attaquer seul en conduite de balle et tirer. Enfin, la figure $15 \mathrm{~b}$ montre une phase de jeu classique avec le porteur de balle au centre du terrain avec un EJE au milieu, la défense étant en barrage. Dans ce cas très courant, la tactique de l'attaque reste à mettre en place.

La disponibilité de ces configurations prototypiques en mémoire permet aux joueurs de construire des prototypes par catégorisation de formes géométriques, de classes de propriétés d'objets et enfin des catégorisations de relations temporelles en vue d'être plus efficaces en jeu. Ces configurations du jeu sont momentanées et se construisent au fil du jeu, elles s'enchaînent les unes après les autres. Néanmoins pour les décrypter, il faut des repères, des images opératives qui permettent de proposer une réponse rapide à la situation perçue. Quelques signes permettent d'identifier rapidement une situation de jeu et son devenir. De ce fait, attirer l'attention des joueurs sur des points clés peut servir avant l'action dans la façon de comprendre, d'organiser, de préparer et de répondre rapidement au besoin du jeu ou du geste. En conséquence des modèles de jeu peuvent être visualisés avec des manipulations d'images susceptibles de faire réfléchir les sujets sur "comment faire». En un mot, comprendre pour réussir. Ces configurations prototypiques, quand elles sont adéquatement construites, constituent une sorte de « prêt à porter décisionnel » pour le joueur lui permettant d'exploiter plus vite les réponses inscrites dans la configuration momentanée qui a été reconnue comme analogue ou très proche du prototype. C'est comme du prêt à utiliser et non pas du sur mesure qui demande plus de temps.

5. 3. Décomposition du jeu dans l'espace

En football, le cas le plus général concernant l'enchaînement des configurations du jeu est le jeu indirect. II correspond à environ $90 \%$ des actions de jeu et peut être subdivisé en deux sous-ensembles en fonction des dimensions principales du terrain. D'une part un jeu plutôt dans la largeur et d'autre part un jeu plutôt dans la profondeur mais qui au final nécessite de ramener la balle de la périphérie vers le centre. Quant au jeu plutôt direct, il peut être spécifié par du jeu dans la profondeur et analysé à partir de deux autres sousensembles : le jeu dans l'espace de jeu direct et le jeu dans le couloir de jeu direct (axe ballon / la cible à tout moment). Ce que l'on appelle le jeu dans l'axe (but attaqué/but défendu) n'est qu'un cas particulier du jeu direct alors que, dans le vocabulaire courant 


\section{eJRIEPS Hors série n¹ Décembre 2015}

concernant le football, on appelle jeu direct ce jeu avec la balle dans l'axe du terrain (Gréhaigne, Billard, \& Laroche, 1999).

Tableau I. Référentiel d'analyse par rapport à l'espace.

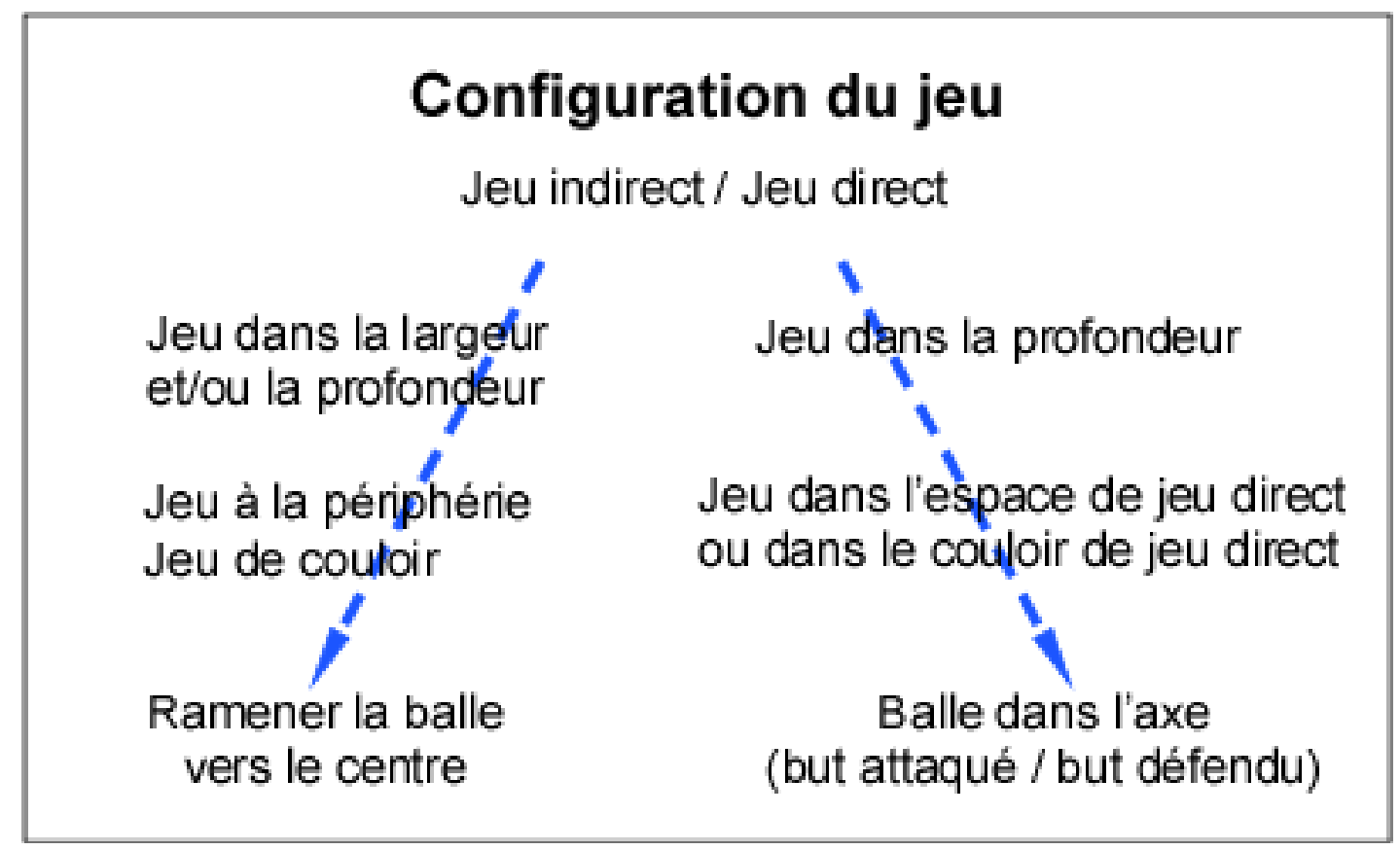

Ce référentiel d'analyse (tableau I) constitue un cadre de référence commun relativement simple pour lire les configurations du jeu et ainsi permettre aux joueurs de combiner les choix tactiques individuels et la nécessaire cohérence de la sommation de ces décisions pour obtenir un jeu construit.

\section{Quelques concepts et actions complémentaires}

Sur le terrain, la simple répartition spatiale des joueurs à différents endroits entraîne, le plus souvent, une répartition non homogène des joueurs impliqués dans la configuration. Le système d'affrontement évolue constamment en sport collectif. Ceci correspond donc, le plus souvent, à une homogénéité de la distribution des joueurs en fonction des rapports de vitesse entre ceux-ci et leurs localisations. Pour tenter de mieux comprendre ces différents états et leurs transformations, nous allons nous intéresser à la genèse du jeu et à son rythme.

\section{1. Phase mère du jeu}

Dans une rencontre de football, le jeu en mouvement au milieu du terrain recèle la logique d'exploitation des situations de déséquilibre, qu'elle tend à faire s'enchaîner "en culbute" (Deleplace, 1979 ; 1994). Une telle filiation semble bien pouvoir être dégagée si, au lieu 


\section{eJRIEPS Hors série n¹ Décembre 2015}

de rester prisonnier de l'ordre chronologique d'apparition des phases dans la séquence de jeu, on part du fait que la logique d'organisation de la première action se retrouve dans toutes les autres, qui ne sont alors que des cas particuliers. Par exemple, en fonction des emplacements de récupération du ballon, cette première action constituera alors la phasemère des autres car elle donnera à l'attaque une forme particulière. Néanmoins, dans une perspective scolaire avec les jeux réduits, il faut souligner que les différentes phasesmères dépendent souvent du niveau du jeu de la rencontre, de l'emplacement de la récupération de la balle et des circulations tactiques qu'elles génèrent. II est d'ailleurs à noter que cette phase d'opposition au milieu de terrain est très fugace dans les jeux à effectifs réduits. Alors, à l'école ou au collège, cette notion de phase-mère du jeu, dont on pressent bien les caractéristiques génériques, se doit d'être étudiée en relation avec les configurations prototypiques.

6. 2. L'offensive, la défensive et les transitions dans le jeu Enchaîner les temps de jeu en défense et en attaque et se préparer à la situation à double effet constitue un maillon important de la tactique collective à propos des configurations du jeu. Dans la défensive, l'aspect statique est constitué par la distribution des joueurs entre les rideaux défensifs. L'aspect dynamique est plus compliqué. L'étude du jeu actuel montre un rideau défensif au front du ballon protégé par un dispositif étagé sur le terrain, soit en fonction du plan de jeu choisi, soit en conséquence d'un rapport de force subi. La couverture défensive axiale et la ré-alimentation des rideaux constituent également des éléments de ce dispositif.

Dans la défensive au football, il faut donc combiner des rideaux étagés dans la profondeur tout en tenant la largeur. La réserve défensive répond au "principe de suppléance continue » du fait de la mise hors de position éventuelle des défenseurs au cours du déroulement de l'attaque. Cette véritable couverture axiale est primordiale car elle empêche le plus souvent les stratégies visant à pénétrer le système défensif dans le couloir central. Elle tend à renvoyer l'attaque à la périphérie (Duprat, 2005), l'obligeant ainsi à se contenter d'une tentative de réalisation différée, ou un enfermement dans l'angle du terrain. Enfin, le gardien de but est toujours en réserve dans l'axe profond. Par son dynamisme, la défense cherche à priver l'attaque d'initiatives. La mise en œuvre de ce choix tactique suppose du dynamisme, de l'agressivité et de la résolution. Elle suppose aussi une très bonne organisation afin que le pressing haut puisse être exploité quand les élèves récupèrent la balle 


\section{eJRIEPS Hors série n¹ Décembre 2015}

Nous présentons figure 16, à l'aide des concepts « en avance » et « en retard » ainsi que " en barrage et/ou à la poursuite " et quand le principe d'égalité des chances est respecté, une illustration des principales actions et configurations de la défensive. L'offensive est aussi illustrée à l'aide des concepts « en avance " et « en retard " ainsi que « pénétrer et/ou contourner ».

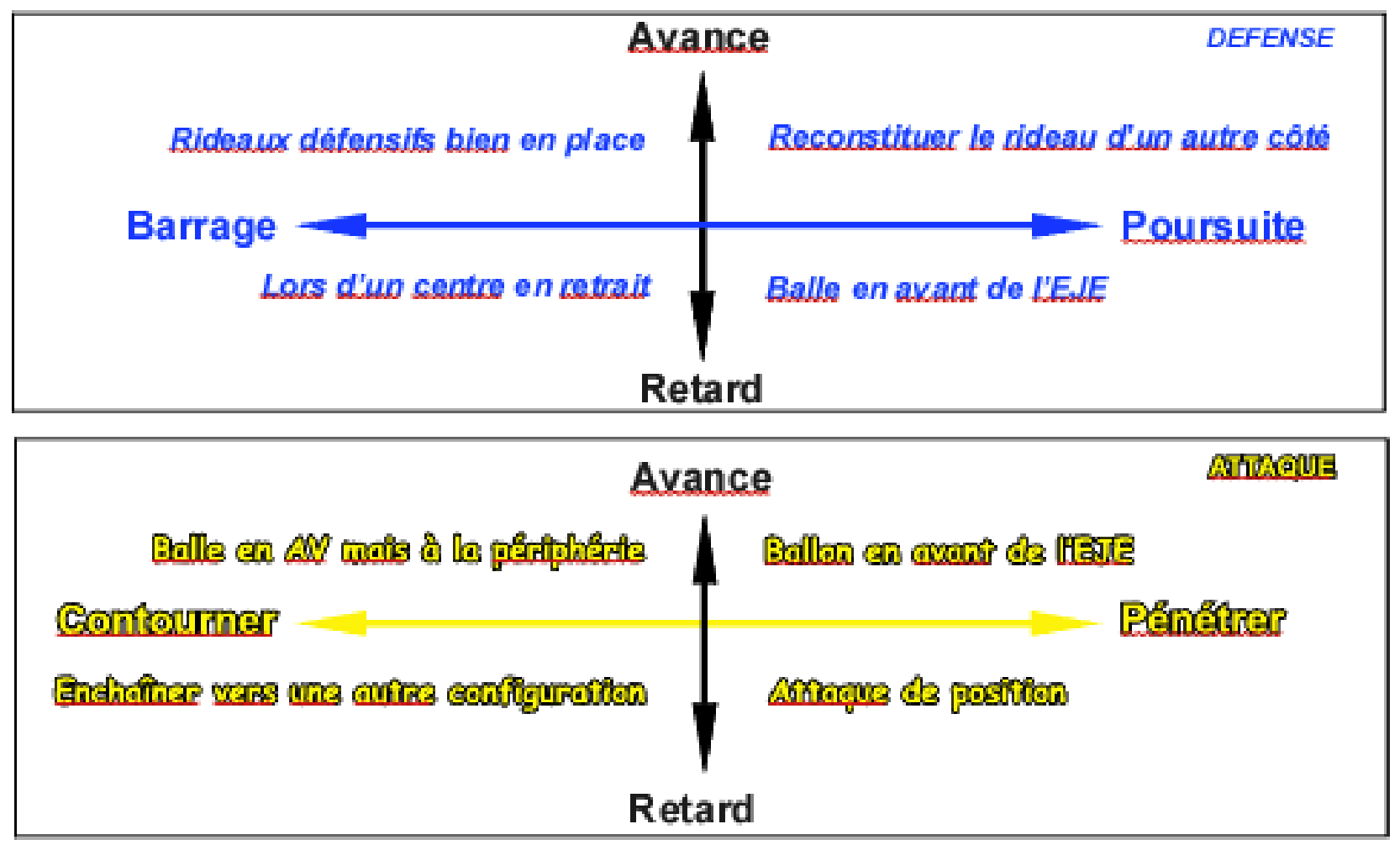

Figure 16. Tâches et enchaînements des temps de jeu pour la défensive et l'offensive.

Quant à elle, l'offensive fonctionne en football, à partir de différentes alternatives : " conduire la balle, s'échanger la balle ou poursuivre un ballon libre". Face à une défense bien en place ou qui s'adapte bien aux offensives menées par l'attaque, celle-ci va augmenter les temps de jeu en tentant de désorganiser le barrage mis en place par la défense. Nous sommes bien dans une " attaque de position» (Wrzos, 1984). Les trajets des joueurs et les trajectoires du ballon à l'attaque consistent alors en des échanges de balle en jeu indirect en relation avec un jeu sans ballon. Cela se concrétise par des démarquages successifs et/ou simultanés des partenaires. La capacité à faire durer les attaques relève néanmoins d'un bon niveau de jeu. Du point de vue de la réversibilité des comportements, il est à noter que dans un rapport de forces défavorable à l'attaque, le fait de faire tourner le ballon à la périphérie de l'EJE peut illustrer l'incapacité de celle-ci à pénétrer le système défensif adverse. 


\section{eJRIEPS Hors série n¹ Décembre 2015}

La réserve offensive, le plus souvent constituée par les partenaires en soutien, est très utile dans la mesure où les partenaires dans la profondeur (en appui) ou à hauteur sont fréquemment dans le triangle d'interception des défenseurs, donc inatteignables. Avec un jeu à la périphérie, qui a toutes les chances de se terminer par un centre en retrait, le "rentré décalé " des partenaires du porteur de balle devient décisif pour une bonne exploitation de cet enchaînement de jeu. Une réserve préventive en cas de perte de balle peut aussi être mise en place.

Dans la pratique sociale de référence ou à partir du 8 contre 8 (Duprat, 2007) pour les jeux à effectif réduit, la situation à double effet (Deleplace, 1966) lie organiquement offensive et défensive en soulignant l'immédiateté du passage d'attaquant à défenseur. Etre capable de passer de la phase offensive à la phase défensive (ou vice et versa), dans un temps très court et à n'importe quel poste en faisant les choix adéquats est un travail important à mener au niveau de l'apprentissage. Quand on est en défense, il faut prévoir déjà un ou des schémas de jeu qui permettent de lancer la contre-attaque dès la récupération du ballon à l'aide de joueurs placés en avant. Si l'on est en attaque, les joueurs placés en soutien, c'est-à-dire en arrière du porteur de balle, doivent aussi considérer dans les actions qu'ils envisagent d'effectuer constituent également la première ligne défensive en cas de perte de balle. Ainsi, dans le jeu en mouvement, les joueurs sont confrontés à l'immédiateté du passage du rôle d'attaquant à celui de défenseur. Cette réversibilité des situations représente un aspect fondamental des sports collectifs en rapport avec le fait que les équipes attaquent et défendent tour à tour, et qu'il y a toujours une part de défense dans l'attaque et une part d'attaque dans la défense. On peut ainsi parler d'une véritable "co-construction » du système attaque / défense, ce système se faisant et se défaisant au gré de l'action. Ce type d'organisation de l'équipe vise, quand on est en défense, à lancer la contre-attaque dès la récupération du ballon à l'aide des joueurs placés en appui. Si l'on attaque, les joueurs en soutien doivent penser qu'ils constituent la première défense contre la contre-attaque en cas de perte du ballon et s'y préparer en conséquence.

La transition dans la situation à double effet est souvent un moment clé, en particulier lors de la récupération du ballon. Dans cette phase, l'ancienne attaque devenue défense doit se réorganiser

- soit s'opposer au front du ballon pour tenter de récupérer immédiatement la balle ;

- soit en recul frein pour avoir le temps de reconstituer les rideaux défensifs ;

- soit si les rideaux sont en place, prête à s'opposer aux pénétrations de l'adversaire. 


\section{eJRIEPS Hors série n¹ Décembre 2015}

Pour la défense devenue attaque, on vise :

- soit une circulation du ballon et des joueurs pour mettre en place une contreattaque ;

- soit en fonction de l'état d'équilibre du système attaque / défense, une rupture momentanée de cet équilibre pour amener un but si l'exécution est rapide;

- soit, en cas d'échec de l'action vers le but, un enchaînement vers d'autres situations avec conservation du ballon pour attendre une autre opportunité.

Concernant les exercices destinés à travailler ce type de configurations du jeu, on a tendance à proposer des situations d'apprentissage avec trop rarement des défenses à la poursuite. Classiquement, on fixe le point de départ de l'attaque avec un nombre de joueurs, une défense en barrage avec un gardien de but ou pas suivant le thème à travailler. Comme variante, il est possible d'envisager le véritable point de départ de la situation jouée, dès l'instant où le ballon est récupéré par la défense, celle-ci devenant l'attaque. Les joueurs sont confrontés de la sorte à des modalités qui sont très proches des véritables conditions du jeu.

6. 3. La vitesse du jeu

Les rapports d'opposition dans le mouvement général évoluent et les séquences de jeu s'engendrent les unes dans les autres. Temps et vitesse deviennent donc des éléments cruciaux de la réussite et le nécessaire cadre de référence à toutes décisions. Lemoine \& Jullien (2004) notent d'ailleurs que dans le football actuel les attaquants sont confrontés à des réseaux défensifs de plus en plus denses où les espaces d'évolution sont réduits. Jouer en déviation apparaît alors comme une solution possible pour modifier le rapport de forces. Néanmoins, beaucoup d'entraîneurs demandent à leurs joueurs de se déplacer rapidement avec le ballon mais ceci ne constitue en aucun cas la meilleure façon d'augmenter la vitesse du jeu. L'augmentation de la vitesse de jeu est très importante pour chaque équipe de football et " en avance » ou " en retard " restent des concepts clés car fondamentalement liés au temps. Cette augmentation de la vitesse du jeu n'est pas à la portée de tous les joueurs et donc de toutes les équipes. Elle constitue en particulier chez les jeunes et dans le milieu scolaire un indicateur fiable de la capacité à élever son niveau de jeu. D'ailleurs, c'est souvent la capacité à fixer rapidement un ou deux défenseurs qui permet l'émergence de solutions en attaque. Pour une bonne analyse des configurations du jeu, l'espace n'est, ma foi, qu'un indicateur commode, concret et plus facile à visualiser pour le débutant. Par contre la vitesse du jeu est un indicateur qui est assez facile à 


\section{eJRIEPS Hors série n¹ Décembre 2015}

décoder pour un observateur extérieur car les séquences de jeu vont s'enchaîner de façon plus nette et surtout plus fréquemment.

\section{4. Régulations et temps}

Dans l'Encyclopaedia Universalis, Canguilhem (2015, p. 01) écrit que

«... le concept de régulation, dans son acception la plus large, renferme au minimum trois idées : celle de relation d'interaction entre éléments instables, celle de critère ou de repère, celle de comparateur. La régulation, c'est l'ajustement, conformément à quelque règle ou norme, d'une pluralité de mouvements ou d'actes ou de leurs effets ou produits que leur diversité ou leur succession rend d'abord étrangers les uns aux autres».

\section{4. 1. Le temps et la réponse}

Dans les configurations complexes, il existe un certain nombre d'aléas pour lesquels le joueur n'a pas immédiatement de réponse toute prête. Le joueur doit donc faire face à une situation de résolution de problème qui l'oblige à rechercher et recourir à des connaissances et compétences motrices issues de sa formation ou de son expérience. Là, deux voies s'offrent à lui : ou bien il s'attache à la seule recherche d'une solution et est satisfait quand il l'a trouvée, ou bien il élabore une stratégie cognitive qui lui permettra ultérieurement de résoudre non seulement ce problème précis posé par la configuration du jeu en cours, mais aussi les problèmes de même type dans d'autres configurations. On retrouve ici des idées déjà postulées par l'apprentissage par analogie.

On dira aussi que dans le premier cas, le joueur cherche une réponse pour résoudre le problème ponctuel. Dans le second cas qu'il cherche une stratégie cognitive pour résoudre les problèmes de la même classe. Cette distinction est identique à la différence que Piaget (1974) faisait entre « réussir » et « comprendre » en déclarant que « réussir » relève du «savoir-faire » et « comprendre » correspond à la conceptualisation, quand cette dernière précède l'action ou bien qu'elle agit en retour sur l'action.

\section{4. 2. Des états dynamiques ou complexion du jeu}

II est possible de définir les micro-états du système attaque / défense momentané par une distribution des joueurs sur le champ de jeu en fonction de leurs positions, leurs orientations et leurs vitesses de déplacement. Une telle distribution dynamique est par définition évolutive. Cette dernière précède l'action ou bien qu'elle agit en retour sur l'action. On revient aux concepts de tactique et stratégie, le second concernant le projet ou plan d'action, le premier se référant à la concrétisation donc à la mise en œuvre, celleci s’appuyant sur les acquis travaillés en amont et doit se traduire par des apprentissages. 


\section{eJRIEPS Hors série n¹ Décembre 2015}

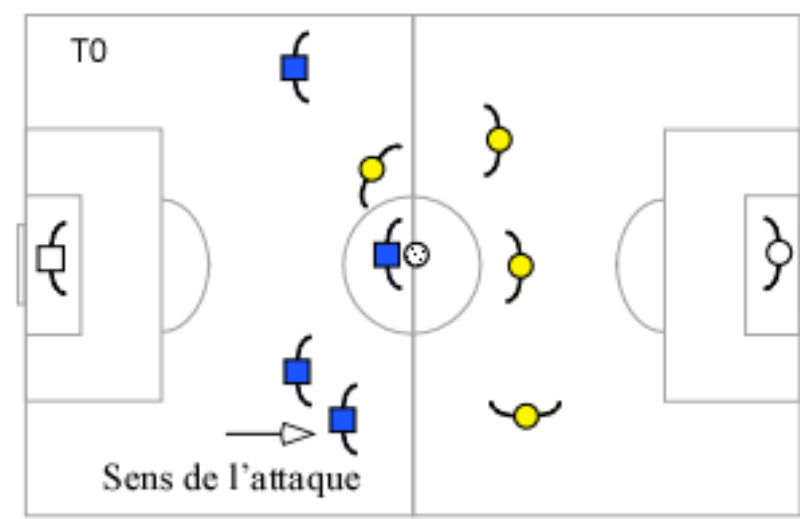

Figure 17a. Configuration à T 0

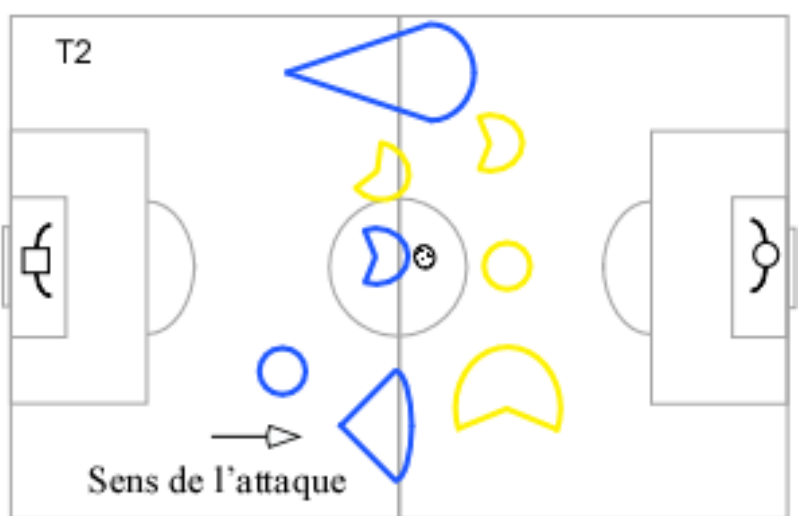

Figure 17b. Complexion à $\mathrm{T}$ plus 2 secondes

Dans le jeu en mouvement, le désordre apparent renvoie souvent à une homogénéité particulière autre que la simple distribution spatiale des joueurs sur le terrain, puisqu'il s'agit d'une distribution sur des niveaux de vitesses. Dans les situations d'opposition, les rapports de vitesse entre les joueurs aboutissent au fait que des états spatialement non homogènes soient compensés et stabilisés. Cela veut dire que ces états apparaîtraient plus homogènes à un observateur qui serait capable de décoder les rapports de vitesse tandis qu'une observation classique mettrait seulement en évidence des aspects hétérogènes mais structurés en rapport avec des positions et des formes géométriques. En référence et par analogie aux concepts utilisés par Planck (1941), une telle distribution constitue ce que nous appellerons "une complexion du système". Les complexions possèdent en elles-mêmes de nombreuses transformations qui sont à la fois restreintes en fonction des possibles évolutions continues du jeu mais importantes si l'on choisit une rupture en modifiant le mouvement en cours. II est évident qu'en deux secondes (figure 17 $a$ et b), avec des joueurs en déplacement, la complexion du jeu a beaucoup évolué. Certains joueurs ont peu bougé mais d'autres, lancés à pleine vitesse, ont donné une expansion considérable à cet état dynamique. Une des caractéristiques de la circulation des joueurs est qu'elle peut être à la fois très rapide mais aussi entrecoupée d'une succession d'arrêts. Les transitions du jeu entre deux états (deux configurations momentanées du jeu) deviennent alors des éléments d'observation essentiels pour des décisions appropriées. Barrage / position et poursuite/ avance deviennent des éléments premiers de la prise d'informations.

Entre le moment où les informations sur l'état du dispositif contrôlé sont saisies par le joueur et le moment où la décision de ce dernier produit son effet, il s'écoule un temps plus ou moins long qui dépend du temps requis par les actions que ce joueur doit exécuter et les caractéristiques des éléments concrets de la configuration. Pendant ce temps la 


\section{eJRIEPS Hors série n¹ Décembre 2015}

configuration peut ou non évoluer... d'où parfois la nécessité d'une nouvelle régulation. Ici, la forme d'intelligence engagée dans la pratique doit combiner à la fois le flair, l'attention vigilante, le sens de l'opportunité, l'anticipation... Dans ce cas, l'accent est toujours mis sur "l'efficacité pratique " en un mot la recherche du succès dans le domaine de l'action. Cette efficacité pratique doit être suffisamment adaptable et déliée dans la mesure où elle est confrontée à des situations constamment changeantes. Elle doit rester ouverte à de nombreux possibles pour en déceler la cohérence et profiler son évolution, en un mot s'appuyer sur le potentiel de la situation (Gréhaigne, Caty, \& Marle, 2004).

6. 5. Un joueur agent de ses propres transformations

Avec ces éléments nous visons un joueur praticien ainsi qu'agent de sa propre formation et capable de jouer tout au long de sa vie. Nous entendons précisément par praticien, un joueur capable d'organiser le jeu mais également capable d'adapter, de réguler et d'évaluer ses actions face à des configurations changeantes et évolutives. Cette autre forme de régulation comprend deux formes principales : l'une au cours du jeu qui consiste à ajuster ses actions, l'autre a posteriori, qui vise à établir un bilan de ce qui s'est passé et à en tirer les conséquences. La régulation tactique au cours du jeu constitue, pour nous, un élément clef du dispositif car elle assure une adéquation permanente entre le système de contraintes proposé par les configurations momentanées du jeu et les ressources dont dispose le joueur. Dans cette optique, un joueur concepteur et praticien, capable d'analyser, de réguler sa pratique ainsi que de reformuler son projet devient de ce fait «agent de sa propre formation»(Lesnes, 1977, p. 178). Ce type de joueur peut établir lui-même une régulation entre sa pratique et des moments de réflexion / analyse sur celleci. Dès lors, le joueur peut construire des règles de gestion de son comportement qui lui permettront d'évoluer et possiblement de poursuivre sa formation tout au long de sa carrière de joueur.

\section{Discussion}

Deux notions se trouvent ainsi au cœur de l'analyse du jeu : d'une part, celle de configuration du jeu telle qu'elle s'actualise et prend forme dans le rapport de force en cours et d'autre part celle de potentiel de situation tel qu'il se trouve impliqué dans la situation et dont on doit tirer parti (Jullien, 1996). Le joueur doit donc prendre appui sur le potentiel inscrit dans la situation pour prendre des décisions tout au long de son évolution. Ainsi, l'idée de prédéterminer le cours des événements en fonction d'un plan que l'on 


\section{eJRIEPS Hors série n¹ Décembre 2015}

aurait dressé à l'avance se trouve en partie exclue. C'est l'évaluation des forces en présence au potentiel qui s'en dégage qui représente un moment clé de la réflexion. $A u$ sein du processus antagoniste, l'interaction est continue. Aussi, c'est en fonction de ce qui est dommageable pour les adversaires à tout moment que le joueur perçoit ce qui lui est profitable et réciproquement.

La tactique repose donc sur les décisions successives, prises en fonction de l'évolution de l'action. Bien exploiter une configuration du jeu renvoie toujours à une ou des analyses pertinentes des propriétés de cette configuration. Ainsi, le joueur pourrait prendre des décisions plus rapides en développant :

- sa capacité de catégorisation de ce qu'il relève dans le réel et qui permet, à la vitesse du jeu, de ramener les configurations à quelques classes de problèmes pour lesquels le joueur dispose d'indices avant-coureurs facilitant ainsi une anticipation ;

- sa connaissance de l'essentiel des réponses motrices individuelles et collectives pour chaque classe de problèmes ainsi que la maîtrise des critères de choix pour les différentes réponses.

Tout cet ensemble ne peut émerger qu'au niveau cognitif où sont possibles les choix et la résolution des événements inattendus. Le développement de l'aptitude tactique suppose le développement de la capacité à décider et à décider vite, laquelle dépend de l'aptitude à concevoir des réponses adaptées.

Aujourd'hui, tout le monde est à peu près d'accord pour convenir que pour bien analyser les sports collectifs, il faut recourir à une approche dynamique du jeu. Le problème est désormais de fournir des concepts, des connaissances et des contenus permettant de soutenir cette affirmation (Menaut, 1998). En poursuivant cette réflexion, nous pouvons dire que les systèmes complexes non linéaires, ou simplement linéaires par morceau, peuvent faire preuve de comportements peu prévisibles qui peuvent même sembler aléatoires. Pour mieux analyser le jeu, un des objectifs importants est la description d'états stationnaires du système. Ce sont des configurations du jeu qui temporairement n'évoluent plus avec le temps. On peut penser que certains de ces points fixes sont attractifs ; cela signifie que si le système parvient à leur voisinage, il va converger vers cet état d'équilibre. On s'intéressera également aux aspects périodiques, c'est-à-dire aux états du système qui se répètent au bout d'un certain temps. Les configurations prototypiques nous semblent un exemple particulièrement probant de ces aspects périodiques (Gréhaigne \& Godbout, 2014). La notion d'état dynamique permet de comprendre davantage qu'à un instant donné, les joueurs sont en mouvement. Ils ont une 


\section{eJRIEPS Hors série n¹ Décembre 2015}

position mais cette position est en train d'évoluer car ils possèdent fréquemment une vitesse instantanée différente. Alors, l'évolution du système complexe ne peut se modéliser qu'en concevant une évolution discontinue dans le temps.

Avec ces modèles dynamiques à disposition il est possible de se pencher sur la tactique individuelle avec quelques règles d'action simples. Pour une bonne tactique individuelle offensive, une évidence est à rappeler : l'équipe qui marque le plus de buts remporte le match. L'attaque commence souvent à l'arrière de l'espace de jeu effectif. Ici, s'appliquer sur les premières passes en relation avec une transition rapide en jouant vite vers l'avant est souvent un gage de réussite. Contrôler la balle en mouvement pour provoquer de l'incertitude chez l'adversaire et savoir où je vais donner la balle avant de la recevoir contribue aussi à accélérer le jeu. Pour les non porteurs de balle proches de l'action, il faut rechercher les intervalles pour proposer une solution au porteur de balle, le tout en mouvement. Enfin, il est nécessaire d'être présent devant le but avec l'envie de marquer ou faire marquer car amener le ballon en avant de l'espace de jeu effectif et dans l'axe central constitue une action de jeu très dangereuse pour la défense. En un mot, toute action qui permet de prendre de l'avance sur le replacement défensif constitue une action positive pour l'attaque et peut amener une action de marque. Le facteur vitesse est à examiner de près car il joue un rôle important chez le joueur de sport collectif en relation avec les accélérations et les décélérations que celui-ci peut produire. Nous y reviendrons plus avant mais de bonnes qualités de vitesse permettent, dans des conditions données, d'accomplir des actions motrices en un laps de temps minimum ou de distancer son adversaire direct. Pour réussir en défense, il devient nécessaire de prendre en compte le placement de ses coéquipiers. Si je suis en infériorité, je ralentis la progression avec un « recul frein » en attendant le retour de mes coéquipiers ; si je suis en supériorité, je fais un pressing sur le porteur de balle. Si je souhaite emmener mon adversaire direct sur ma gauche, je me place de trois quart avec mon pied droit devant. II faut penser à orienter le joueur du côté de la ligne de touche car celle-ci est un allié pour le défenseur ou orienter l'adversaire vers l'intérieur du terrain, pour l'emmener là où la densité de co-équipiers est plus importante. Ce choix dépend en partie du temps dont on dispose pour agir. Que le duel soit offensif ou défensif, le joueur qui dispose de la plus grande capacité de vitesse possède un énorme avantage et en plus, il a toujours la possibilité de varier la vitesse de réalisation durant l'action pour mieux surprendre les joueurs adverses. Au plan de la motricité, une bonne vitesse est étroitement liée à la vitesse de réaction et à une bonne accélération sur les premiers mètres. Au plan de la tactique individuelle, la rapidité de 


\section{eJRIEPS Hors série n¹ Décembre 2015}

perception (enregistrer, traiter et évaluer au moyen des sens les informations essentielles sur le déroulement du jeu), la rapidité d'anticipation (prévoir les actions des adversaires/coéquipiers ainsi que l'évolution du jeu) et la vitesse de décision (se décider le plus vite possible pour une action effective) sont aussi des facteurs de succès.

Enfin, l'analogie perçue entre deux ou plusieurs configurations du jeu est à analyser très finement. Les ressemblances permettent d'organiser en mémoire les événements dans des catégories familières. Ainsi, pour gagner du temps quand on passe d'un jeu à un autre, le processus qui permet de comprendre une nouvelle situation de jeu doit être conçu en termes de rapport avec ce qui est déjà en mémoire. Alors, l'analogie entre deux situations de jeu peut fournir des systèmes de référence pour traiter promptement ces nouvelles configurations perçues du jeu (Zerai, Gréhaigne, \& Godbout, 2013).

\section{Conclusion}

Ces différentes analyses n'ont d'intérêt que si elles débouchent sur du concret, en améliorant l'efficacité en jeu, d'où la focalisation sur la variété des apprentissages liés aux systèmes de contraintes qui s'exercent sur le joueur au cœur de l'action. Elles doivent permettre de dégager, à partir d'invariants, des propriétés du jeu et du comportement de l'équipe, les règles essentielles pour agir sur l'affrontement. Ainsi, le comportement intelligent d'une équipe sera le résultat de l'activité cognitive et motrice des joueurs qui vise à la résolution des problèmes posés par la mise en relation des perturbations issues du fonctionnement des différents sous-systèmes et des choix effectués pour compenser et / ou accentuer ces déséquilibres. Dans cette perspective, l'erreur tactique n'est pas seulement le résultat d'une perturbation non compensée mais la conséquence de conduites probabilistes qui n'ont pas fonctionné dans cette trame toujours mouvante que constitue le jeu.

Au cours de cet article, nous avons été amenés à parler de construction du joueur, des savoirs, etc. Chacun de nous génère ses propres règles et ses schémas de pensée qu'il utilise pour donner du sens à son expérience. Ce cadre de références nous semble extrêmement fécond pour la didactique des sports collectifs et nous aurons bientôt l'occasion d'y revenir pour approfondir son apport à la recherche en éducation physique.

Les solutions formelles capables de résoudre un problème posé par le jeu peuvent être nombreuses. Pourtant chaque solution constitue un peu une entité propre à un contexte toujours singulier. Ensuite, il faut admettre que toute modélisation représente comme nous venons de le voir, un nécessaire appauvrissement de la grande variété des données issue 


\section{eJRIEPS Hors série n¹ Décembre 2015}

de la pratique de référence, d'où la tentation de réduire les propriétés du jeu à quelques préceptes... Enfin de nombreux phénomènes liés à la communication et à la tactique résistent aux tentatives de formalisation. Dans les sports collectifs où l'on tient le ballon (handball ou basket-ball par exemple), des caractères particuliers apparaissent car le jeu sur un espace stabilisé prend relativement de l'importance. Dans ce cas, le perfectionnement chez les élèves passe souvent par la construction d'un code de la circulation des joueurs et de la balle. II s'agira donc de faire construire ou de fournir aux élèves des modèles du jeu à partir de quelques configurations prototypiques relativement stables.

Enfin, ce type de réflexion et d'analyse devrait permettre de passer d'une conception statique à une conception plus dynamique de la trame de transformation que constituent les rapports d'opposition dans une rencontre de sport collectif. Cela obligera, sans doute, d'évoluer d'une centration quasi exclusive sur la circulation du ballon à une vision plus globale de la situation d'affrontement prenant en compte les trajectoires de la balle certes mais aussi les déplacements, la vitesse et l'orientation des joueurs engagés dans la configuration momentanée du jeu.

Le rapport direct à l'opposition et la nécessité de sortir des techniques académiques pour adapter sa motricité aux diverses contraintes font que les sports collectifs sont des outils exceptionnels pour développer une activité adaptative élargie. Parfois quand la coordination motrice n'est pas au rendez-vous, le joueur est exposé à des pertes de balle. Néanmoins, l'intelligence tactique grâce à un déplacement et/ou un placement pertinent permet de disposer de plus de temps pour produire un geste juste engendrant ainsi une succession de transformations.

\section{Bibliographie}

Ali, A.H., \& Farraly, M. (1990). An analysis of patterns of play in soccer. Science and Football, 3, 37-44.

Bailly, B. (2004). Conscience de la situation des conducteurs aspects fondamentaux, méthodes et application pour la formation des conducteurs. Thèse de Doctorat (non publiée) en psychologie cognitive. Université Lumière Lyon 2.

Bisseret, A. (1995). Représentation et décision experte, psychologie cognitive de la décision experte chez les aiguilleurs du ciel. Toulouse : Octarès.

Canguilhem, G. (2015). Régulation, épistémologie. Encyclopædia Universalis [en ligne]. URL : http://www.universalis.fr/encyclopedie/regulation-epistemologie/

Caty, D., \& Gréhaigne, J.F. (2005). Modélisations de l'attaque et didactique des sports collectifs en EPS. . e Journal de la Recherche sur l'Intervention en Education Physique et sportive, 8, 75-88. Besançon : IUFM de Franche-Comté.

Caty, D., Meunier, J.N., \& Gréhaigne, J.F. (2007). Modélisations des attaques réussies pour progresser dans les sports collectifs en EPS. Spirales, 40, 105-116.

Deleplace, R. (1966). Le rugby. Paris : Colin-Bourrelier. Dugrand 


\section{eJRIEPS Hors série n¹ Décembre 2015}

Deleplace, R. (1979). Rugby de mouvement-Rugby total. Paris : Éducation Physique et Sports.

Deleplace, R. (1983). La recherche sur la spécialisation sportive, l'entraînement, la performance. Actes du colloque "La recherche en S.T.A.P.S.", Nice, 19-20 septembre 1983, 93-151.

Deleplace, R. (1994). La notion de matrice d'action pour les actions motrices complexes. In D. Bouthier \& J. Griffet (Eds.) Représentation et action en activité physique et sportive (pp. 25-42). Université de Paris-Orsay.

Dietsch, G. (2014). Le modèle du « futsal » en EPS : une forme de pratique scolaire du football en milieu difficile. Mémoire de mastère (non publié). Université Paris-Est Créteil.

Dietsch, G. (2015). Approche technologique et forme de pratique scolaire du football en milieu difficile. $e$ Journal de la Recherche sur l'Intervention en Education Physique et sportive, 35, 60-85.

Dugrand, M. (1989). Le football : de la transparence à la complexité. Paris : PUF.

Duprat, E. (2005). Approche technologique de la récupération du ballon lors de la phase défensive en football, contributions à l'élaboration de contenus de formation innovants. Thèse de Doctorat. ENS Cachan.

Duprat, E. (2007). Enseigner le football en milieu scolaire (collèges ou lycées) et au club. Editions Actio, Paris.

Edelman, G. (1992). Bright air, brilliant fire: On the matter of the mind. New York : Basic Books.

Edelman, G., \& Mountcastle, V.B. (1978). The midful brain. Cambridge : M.I.T. Press.

Gréhaigne, J.-F. (1986). Quantité et qualité de l'information chez différents niveaux de joueurs. Rapport de recherche au Laboratoire d'Etudes et de Recherches sur la Performance Sportive. Université de Bourgogne

Gréhaigne, J.F. (Ed.). (2007). Configurations du jeu, débat d'idées et apprentissage des sports collectifs. Besançon: Presses de l'Université de Franche-Comté.

Gréhaigne, J.F. (Ed.). (2009). Autour du temps. Espaces, apprentissages, projets dans les sports collectifs. Besançon: Presses de l'Université de Franche-Comté.

Gréhaigne, J.-F., \& Caty, D. (2014). Développer la pensée tactique à l'école. In J.-F. Gréhaigne (Ed.) L'intelligence tactique. Des perceptions aux décisions tactiques en sports collectifs (pp. 279-300). Besançon : Presses de l'Université de Franche-Comté.

Gréhaigne, J.-F., \& Godbout, P. (2012). À propos de la dynamique du jeu ... en football et autres sports collectifs. . e Journal de la Recherche sur l'Intervention en Education Physique et sportive, 26, 130-156.

Gréhaigne, J.-F., \& Godbout, P. (2014). Dynamic systems theory and team sport coaching. Quest, 66 (1), 96-116.

Gréhaigne, J.F., Billard, M., \& Laroche, J.Y. (1999). L'enseignement des jeux sportifs collectifs à l'école. Conception, construction, évaluation. Bruxelles : De Boeck.

Gréhaigne, J.F., Bouthier, D., \& David, B. (1997). Dynamic systems analysis of the opponent relationships in the collective actions in soccer. Journal of Sports Sciences, 15, 137-149.

Gréhaigne, J.F., Caty, D., \& Marle, P. (2004). L'apport de la notion de configuration du jeu à la didactique des sports collectifs. In G. Carlier (Ed.), Si l'on parlait du plaisir d'enseigner l'éducation physique (pp. 167-179). Montpellier : AFRAPS.

Gréhaigne, J-F., Godbout, P., \& Zerai, Z. (2014). Analogies, configurations du jeu et intelligence tactique. In J.-F. Gréhaigne (Ed.) L'intelligence tactique. Des perceptions aux décisions tactiques en sports collectifs (pp. 301-314). Besançon: Presses de l'Université de Franche-Comté.

Gréhaigne, J.-F., Marle, P., \& Zerai, Z. (2013). Modèles, analyse qualitative et configurations prototypiques dans les sports collectifs. eJRIEPS, 30, 05-25

Hemphill, D. (2005). Deeper Inside the Beautiful Game. Journal of The Philosophy of Sport, XXXII, 105-115.

Le Boterf, G (1999). De la compétence. Essai sur un attracteur étrange. Les Editions d'organisation.

Jullien, F. (1996). Traité de l'efficacité. Paris : Grasset.

Lemoine, A., \& Jullien, H. (2004). Etude de la production d'Information dans le cadre de la transmission instantanée du ballon en football. e Journal de la Recherche sur l'Intervention en Education Physique et sportive, 6, 47-55.

Lesnes, M. (1977). Travail pédagogique et formation d'adulte : éléments d'analyse. Paris : Presses Universitaires de France.

Mahlo, F. (1969). Acte tactique en jeu. Paris : Vigot.

McMorris, T. \& Graydon, J. (1997). The contribution of the research literature to the understanding of decision making in team games. Journal of Human Movement Studies, 33, 69-90.

McPherson, S.L. (1993). Knowledge representation and decision making in sport. In J.J. Starkes and F. Allard (Eds.) Cognitive issues in motor expertise (pp. 159-188). Elsevier Science Publishers B.V.

Ochanine, D. A. (1969). Rôle de l'image opérative dans la saisie du contenu informationnel des signaux. Questions de Psychologie, 4, 209-224. 


\section{eJRIEPS Hors série n¹ Décembre 2015}

Ochanine, D. (1978). Le rôle des images opératives dans la régulation des activités de travail. Psychologie et Education, 2, 63-72.

Paillard, J. (1987). Système nerveux et fonctions d'organisation. In J. Piaget, J.P., Bronckart, \& P. Mounoud (Eds.), La Psychologie (pp. 1378-1441).Paris : Gallimard, Encyclopédie de la Pléïade.

Perrenoud, P (1999). Construire des compétences, tout un programme. Vie pédagogique, 112, 16-20.

Piaget, J. (1974). Réussir et comprendre. Paris : PUF.

Planck, M. (1941). Initiation à la physique. Paris: Flammarion.

Spérandio, J. C. (1984). L'ergonomie du travail mental. Paris.

Wrzos,J. (1984). La tactique de l'attaque. Bräkel : Broodcoorens.

Zerai, Z., Gréhaigne, J.-F., \& Godbout, P. (2013). Configurations of play in invasion team sports and learning by analogy. International Journal of Physical Education Volume L (1) 18-28. 